Interfaces and Free Boundaries 14 (2012), 105-143

DOI 10.4171/IFB/275

\title{
Asymptotic analysis for Korteweg models
}

\author{
WOLFGANG DREYER \\ Weierstrass Institute for Applied Analysis and Stochastics, Berlin, Germany \\ dreyer@wias-berlin.de \\ JAN GIESSELMANN \\ University of Stuttgart (IANS), Stuttgart, Germany \\ giesselmann@ians.uni-stuttgart.de \\ Christiane Kraus \\ Weierstrass Institute for Applied Analysis and Stochastics, Berlin, Germany \\ kraus@wias-berlin.de \\ CHRISTIAN ROHDE \\ University of Stuttgart (IANS), Stuttgart, Germany \\ rohde@ians.uni-stuttgart.de
}

[Received 5 October 2010 and in revised form 20 October 2011]

\begin{abstract}
This paper deals with a sharp interface limit of the isothermal Navier-Stokes-Korteweg system. The sharp interface limit is performed by matched asymptotic expansions of the fields in powers of the interface width $\varepsilon$. These expansions are considered in the interfacial region (inner expansions) and in the bulk (outer expansion) and are matched order by order. Particularly we consider the first orders of the corresponding inner equations obtained by a change of coordinates in an interfacial layer. For a specific scaling we establish solvability criteria for these inner equations and recover the results within the general setting of jump conditions for sharp interface models.
\end{abstract}

2010 Mathematics Subject Classification: Primary 76T10; Secondary 35C20, 35R35.

Keywords: Liquid vapor flow; phase transition; asymptotic analysis; sharp interface limit.

\section{Introduction}

Phase transitions in single substance flows are usually described by two kinds of models: the sharp interface model and the diffuse phase field model. The conventional and physically more intuitive approach is the sharp interface model. In the sharp interface approach, interfaces separating the coexisting phases or structural domains are modeled as hypersurfaces at which certain quantities such as the density or the pressure suffer jump conditions. Local quantities at the interface are then determined from the boundary conditions or are calculated from the driving force for interfacial motion. The structure of possible interfacial conditions is quite well-founded from the view point of thermodynamics. However from the numerical point of view the sharp interface approach involves the explicit tracking of the interface and becomes often numerically impractical for complicated microstructures. Because of these disadvantages, the phase field approach has emerged as a powerful method during the last twenty years. A phase field model represents a microstructure, both the compositional domains and the interfaces, as a whole. The interface between different phases is described by a small transition region, where an order parameter, representing the phases, changes 
its state smoothly. The microstructural evolution is modeled by a system of partial differential equations. The phase field model contains the corresponding sharp interface description as a particular limit, i.e., if the interfacial thickness tends to zero. An overview about a large class of phase field models and their sharp interface limits can be found in [2]. For phase field models it is of overall interest to validate them, by investigating the sharp interface limit in appropriate scaling regimes.

In this work we consider a sharp interface limit of the isothermal Navier-Stokes-Korteweg phase field model for a particular scaling. The sharp interface limit is performed by matched asymptotic expansions of the fields of these models in powers of the interface width $\epsilon$. These expansions are considered in the interfacial region (inner expansion) and in the bulk (outer expansion), and are matched order by order. This results in partial differential equations for the diffuse field and a series of boundary conditions at the interface from which we achieve jump conditions in the sharp interface limit.

We consider the case where the Mach number is of order $\mathcal{O}(1)$ and viscosity and capillarity are both of order $\Theta\left(\varepsilon^{2}\right)$. Our particular scaling of the viscosity leads to a no-entropy-dissipation kinetic relation in the leading order $\mathcal{O}(1)$. The resulting sharp interface model is the isothermal Euler model. In the first order $\mathcal{O}(\varepsilon)$ we obtain a Young-Laplace law and a non-zero entropy dissipation, which is determined by the viscosity. The fact that the surface tension is of order $O(\varepsilon)$ is in agreement with the results obtained in $[9,20,21]$ for equilibria. A different kinetic relation was considered in [5], there surface tension and entropy dissipation are of order $\mathcal{O}(1)$ and $\mathcal{O}(\varepsilon)$ respectively. There are also other physically meaningful scalings, for instance the case where the viscosity is of order $\mathcal{O}(\varepsilon)$, which implies entropy dissipation already in the leading order [3,16,23]. Another possibility is to look at a low Mach number limit linked to the sharp interface limit, which can be found in [15], and in $[17,18,22]$ for the one phase case.

The paper is organized as follows: We start with describing the Navier-Stokes-Korteweg phase field model in Section 2. The corresponding sharp interface setting is introduced in Section 3. By non-dimensionalization we introduce the smallness parameter $\varepsilon$ with physical interpretation, see Section 4. In Section 5 we introduce the asymptotic expansions and provide formulas for the geometric properties of the interface in two dimensions. Then we state our main results in Section 6, which are proven in Sections 7 and 8 respectively. In Section 9 we investigate the kinetic relation comprised in the jump conditions derived in Section 6. We show that the state on one side of the phase boundary already determines the interfacial velocity and the state on the other side of the phase boundary. Furthermore this property does not follow from the jump conditions for mass and momentum alone.

\section{The Navier-Stokes-Korteweg phase field model}

We consider an isothermal, compressible fluid with density $\rho \in(0, b)$ and velocity $\mathbf{u} \in \mathbb{R}^{d}$, that is capable to undergo phase transitions. We assume that the stress tensor can be additively decomposed according to

$$
\sigma=\sigma_{N S}+\sigma_{K},
$$

where $\sigma_{N S}$ denotes the classical Navier-Stokes stress and $\sigma_{K}$ is called Korteweg stress that takes care of possible phase transitions. The Navier-Stokes stress has the form

$$
\sigma_{N S}:=\lambda(\operatorname{div} \mathbf{u}) I+\mu\left(\nabla \mathbf{u}+(\nabla \mathbf{u})^{T}\right),
$$



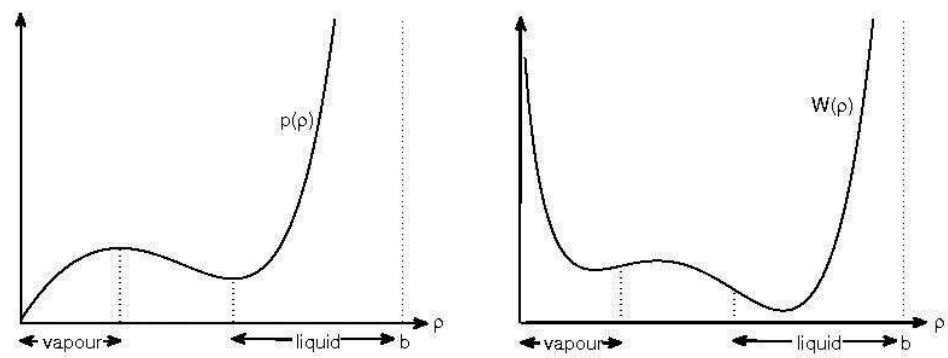

FIG. 1. Van-der-Waals pressure and free energy density function

where $\lambda$ is the bulk viscosity and $\mu$ is the shear viscosity, which satisfy $\mu>0, \lambda+\frac{2 \mu}{d} \geqslant 0$, where $d$ is the space dimension. The Korteweg tensor is given by

$$
\sigma_{K}:=\left(\rho \gamma \Delta \rho+\frac{1}{2} \gamma|\nabla \rho|^{2}\right) I-(\gamma \nabla \rho \otimes \nabla \rho),
$$

where $\gamma$ is a constant which models capillarity effects. The evolution of the model is described by the isothermal Navier-Stokes-Korteweg equations

$$
\begin{array}{rlrl}
\rho_{t}+\operatorname{div}(\rho \mathbf{u}) & =0, & \\
(\rho \mathbf{u})_{t}+\operatorname{div}(\rho \mathbf{u} \otimes \mathbf{u})+\nabla p(\rho) & =\operatorname{div} \boldsymbol{\sigma}_{N S}+\operatorname{div} \boldsymbol{\sigma}_{K}, & & \text { in } \mathbb{R}^{d} \times(0, T) .
\end{array}
$$

We assume that the local part of the pressure $p=p(\rho)$ is a non-monotone function of the density given by a van-der-Waals law. From the density the phase can be directly derived, see Figure 1 . The corresponding Helmholtz free energy density function $W(\rho)$ is related to the pressure by

$$
p(\rho)=\rho W^{\prime}(\rho)-W(\rho)
$$

and has the following properties:

- $W \in C^{2}((0, b),[0, \infty))$,

- $\exists a_{1}, a_{2} \in(0, b): W^{\prime \prime}>0$ in $\left(0, a_{1}\right) \cup\left(a_{2}, b\right), W^{\prime \prime}<0$ in $\left(a_{1}, a_{2}\right)$,

- $\lim _{\rho \rightarrow b} W(\rho)=\infty$.

We will denote the Gibbs free energy by $g$, which is given by $g(\rho)=W^{\prime}(\rho)$.

Smooth solutions of (2.3), (2.4) identically satisfy the entropy inequality, which is given by

$$
\begin{aligned}
\left(W(\rho)+\frac{\rho}{2}|\mathbf{u}|^{2}+\frac{\gamma}{2}|\nabla \rho|^{2}\right)_{t}+\operatorname{div}\left(\left(W(\rho)+\frac{\rho}{2}|\mathbf{u}|^{2}+\frac{\gamma}{2}|\nabla \rho|^{2}\right) \mathbf{u}\right) & \\
+\operatorname{div}\left(\left(p(\rho) I-\boldsymbol{\sigma}_{N S}-\boldsymbol{\sigma}_{K}\right) \mathbf{u}+\gamma \rho \nabla \rho(\nabla \cdot \mathbf{u})\right) & =-\sigma_{N S}:(\nabla \mathbf{u}) \leqslant 0 .
\end{aligned}
$$

An alternative form of the inequality is derived in [1,9]. The existence of classical solutions of (2.3), (2.4) was studied in $[14,19]$, while weak solutions were investigated in $[6,8,11]$. 


\section{The Euler-Korteweg sharp interface model}

Now we describe the phase transition by means of a sharp interface model. In this paper we treat the case where the capillarity $\gamma$ and the viscosities $\lambda$ and $\mu$ are of the same small order. The corresponding sharp interface model is described by the Euler-Korteweg system. In this context the conservation laws of mass and momentum read in the bulk phases $\Omega^{-}(t):=\left\{\mathbf{x} \in \mathbb{R}^{d}: \rho(t, \mathbf{x}) \in\right.$ $\left.\left(0, a_{1}\right)\right\}$ and $\Omega^{+}(t):=\left\{\mathbf{x} \in \mathbb{R}^{d}: \rho(t, \mathbf{x}) \in\left(a_{2}, b\right)\right\}, t \in[0, T)$ :

$$
\begin{aligned}
\rho_{t}+\operatorname{div}(\rho \mathbf{u}) & =0, \\
(\rho \mathbf{u})_{t}+\operatorname{div}(\rho \mathbf{u} \otimes \mathbf{u})+\nabla p & =0 .
\end{aligned}
$$

The interface between two adjacent phases $\Omega^{ \pm}$is described by a moving hypersurface $\Gamma(t)$, i.e., a sharp interface of zero thickness. Across $\Gamma(t)$ the bulk quantities may have discontinuities. We are particularly interested in the case that the interface itself is equipped with mass, momentum, energy and entropy. The jump conditions for these quantities rely on the conservation laws for mass, momentum and energy and on the entropy inequality across the interface $\Gamma$. To state these jump conditions we will use the following notation for some quantity $\psi$ having a jump at the interface. By $\psi^{ \pm}$we denote the limit at the interface from $\Omega^{ \pm}$and we abbreviate

$$
\llbracket \psi \rrbracket:=\psi^{+}-\psi^{-}, \quad\{\psi\}:=\frac{\psi^{+}+\psi^{-}}{2} .
$$

For more details of the following equations we refer to [10].

The general forms of conservation of mass and momentum read:

$$
\begin{aligned}
\llbracket \rho\left(u_{v}-w_{v}\right) \rrbracket & =-\frac{\partial \rho_{\Gamma}}{\partial t}-\left(\operatorname{div}_{\Gamma}\left(w_{t}\right)-\kappa w_{v}\right) \rho_{\Gamma}, \\
\llbracket \rho\left(u_{v}-w_{v}\right)(\mathbf{u}-\mathbf{w})+p v \rrbracket & =-\rho_{\Gamma} \frac{\partial \mathbf{w}}{\partial t}+\operatorname{div}_{\Gamma}\left(\sigma_{\Gamma}\right) .
\end{aligned}
$$

The newly introduced quantities are the normal component of the fluid velocity $u_{v}$, the interfacial mass density $\rho_{\Gamma}$, the interfacial velocity $\mathbf{w}$ which may be decomposed into normal- and tangential speed, i.e., $\mathbf{w}=w_{t} \mathbf{t}+w_{\nu} \boldsymbol{v}$ and $\sigma_{\Gamma} \in \mathbb{R}^{d \times(d-1)}$ is the surface stress tensor. Each surface point is equipped with a tangential matrix $\mathbf{t}$ and a normal vector $\boldsymbol{v}$ and $\kappa$ denotes the sum of the principal curvatures.

We consider exclusively isothermal processes at the temperature $T_{0}$, and these are guaranteed by corresponding heat fluxes $\boldsymbol{q}, \boldsymbol{q}_{\Gamma}$ in the bulk and on the interface. The conservation of energy serves to eliminate the heat fluxes in the entropy inequality, which hereafter becomes the relevant inequality for the isothermal Euler-Korteweg model.

The general form of conservation of energy across the interfaces reads:

$$
\begin{aligned}
& \llbracket \rho\left(u_{v}-w_{v}\right)\left(e+\frac{1}{2}|\mathbf{u}|^{2}\right)+\boldsymbol{q} \cdot \boldsymbol{v}+u_{\nu} p \rrbracket= \\
& \quad-\frac{\partial \rho_{\Gamma}\left(e_{\Gamma}+\frac{1}{2}|\mathbf{w}|^{2}\right)}{\partial t}-\left(\operatorname{div}_{\Gamma}\left(w_{t}\right)-\kappa w_{\nu}\right) \rho_{\Gamma}\left(e_{\Gamma}+\frac{1}{2}|\mathbf{w}|^{2}\right)-\operatorname{div}_{\Gamma}\left(\boldsymbol{q}_{\Gamma}-\mathbf{w}^{T} \boldsymbol{\sigma}_{\Gamma}\right) .
\end{aligned}
$$

The internal energy densities of bulk and interface are denoted by $\rho e$ and $\rho_{\Gamma} e_{\Gamma}$, respectively. 
Finally we give the entropy inequality across the interface. In [1,9] it is shown that the sharp interface version of the Navier-Stokes-Korteweg phase field model has entropy fluxes in the bulk and at the interface that are given by $\boldsymbol{q} / T_{0}$ and $\boldsymbol{q}_{\Gamma} / T_{0}$, respectively. In this case the entropy inequality across the interface reads

$$
\llbracket \rho\left(u_{v}-w_{\nu}\right) s+\frac{\boldsymbol{q} \cdot \boldsymbol{v}}{T_{0}} \rrbracket \geqslant-\frac{\partial \rho_{\Gamma} s_{\Gamma}}{\partial t}-\left(\operatorname{div}_{\Gamma}\left(w_{t}\right)-\kappa w_{\nu}\right) \rho_{\Gamma} s_{\Gamma}-\operatorname{div}_{\Gamma}\left(\frac{\boldsymbol{q}_{\Gamma}}{T_{0}}\right),
$$

where $\rho s$ is the entropy density of the bulk, and the entropy density of the interface is denoted by $\rho_{\Gamma} s_{\Gamma}$.

Next we multiply the momentum balance (3.4) by $\mathbf{w}$ and subtract it from the energy law (3.5). The result is simplified by means of the jump condition for the mass, leading to the general form of the jump condition for the internal energy

$$
\begin{aligned}
\llbracket \rho\left(u_{v}-w_{v}\right)(e+ & \left.\frac{p}{\rho}+\frac{1}{2}|\mathbf{u}-\mathbf{w}|^{2}\right)+\boldsymbol{q} \cdot \boldsymbol{v} \rrbracket \\
& \quad-\frac{\partial \rho_{\Gamma} e_{\Gamma}}{\partial t}-\left(\operatorname{div}_{\Gamma}\left(w_{t}\right)-\kappa w_{\nu}\right) \rho_{\Gamma} e_{\Gamma}-\operatorname{div}_{\Gamma}\left(\boldsymbol{q}_{\Gamma}\right)+\nabla_{\Gamma}(\mathbf{w}): \boldsymbol{\sigma}_{\Gamma}^{T} .
\end{aligned}
$$

We are interested here in a special case where the constitutive law for the surface stress vector $\sigma_{\Gamma}$ has a simple structure:

$$
\sigma_{\Gamma}=\gamma_{\Gamma} \frac{\mathbf{t}}{|\mathbf{t}|^{2}} .
$$

The quantity $\gamma_{\Gamma}$ is called surface tension. In this case the jump condition for the internal energy assumes the special form

$$
\llbracket \rho\left(u_{v}-w_{v}\right)\left(e+\frac{p}{\rho}+\frac{1}{2}|\mathbf{u}-\mathbf{w}|^{2}\right)+\boldsymbol{q} \cdot \boldsymbol{v} \rrbracket=-\frac{\partial \rho_{\Gamma} e_{\Gamma}}{\partial t}-\left(\rho_{\Gamma} e_{\Gamma}-\gamma_{\Gamma}\right)\left(\operatorname{div}_{\Gamma}\left(w_{t}\right)-\kappa w_{\nu}\right) .
$$

Finally we multiply the entropy inequality by $T_{0}$ and subtract it from the jump condition for the internal energy. There follows the interfacial inequality for the Helmholtz free energy $W_{\Gamma}=$ $\rho_{\Gamma} e_{\Gamma}-T_{0} \rho_{\Gamma} s_{\Gamma}$ :

$$
\begin{aligned}
& \frac{\partial W_{\Gamma}}{\partial t}-\left(\gamma_{\Gamma}-W_{\Gamma}\right)\left(\operatorname{div}_{\Gamma}\left(w_{t}\right)-\kappa w_{v}\right) \\
& +\left\{\rho\left(u_{v}-w_{v}\right)\right\} \llbracket \llbracket W^{\prime}(\rho)+\frac{1}{2}|\mathbf{u}-\mathbf{w}|^{2} \rrbracket+\llbracket \rho\left(u_{v}-w_{v}\right) \rrbracket\left\{W^{\prime}(\rho)+\frac{1}{2}|\mathbf{u}-\mathbf{w}|^{2}\right\} \leqslant 0 .
\end{aligned}
$$

Among the objectives of this study is to deduce the Euler-Korteweg sharp interface model from the Navier-Stokes-Korteweg phase field model. Note that the sharp interface model must fit into the setting given here, i.e., we have to recover (3.3), (3.4) and (3.10).

\section{Non-dimensionalization}

We introduce the following reference quantities $x_{r}, t_{r}, u_{r}, \rho_{r}, p_{r}, \mu_{r}$ and $\gamma_{r}$ such that

$$
\mathbf{x}=x_{r} \mathbf{x}^{*}, t=t_{r} t^{*}, \mathbf{u}=u_{r} \mathbf{u}^{*}, \rho=\rho_{r} \rho^{*}, p=p_{r} p^{*}, \lambda=\lambda^{*} \mu_{r}, \mu=\mu^{*} \mu_{r}, \gamma=\gamma_{r} \gamma^{*} .
$$


Then we may rewrite the Navier-Stokes-Korteweg equations (2.3), (2.4) as

$$
\begin{aligned}
\frac{x_{r}}{u_{r} t_{r}} \rho_{t^{*}}^{*}+\operatorname{div}^{*}\left(\rho^{*} \mathbf{u}^{*}\right) & =0, \\
\frac{t_{r} u_{r}}{x_{r}}\left(\rho^{*} \mathbf{u}^{*}\right)_{t^{*}}+\frac{t_{r}^{2} u_{r}^{2}}{x_{r}^{2}} \operatorname{div}^{*}\left(\rho^{*} \mathbf{u}^{*} \otimes \mathbf{u}^{*}\right)+\frac{t_{r}^{2} u_{r}^{2}}{x_{r}^{2} M^{2}} \nabla^{*} p^{*} & =\frac{t_{r}^{2} u_{r}^{2}}{x_{r}^{2} \operatorname{Re}} \operatorname{div}^{*} \sigma_{N S}^{*}+C \gamma^{*} \operatorname{div}^{*} \sigma_{K}^{*},
\end{aligned}
$$

where $M$ denotes the Mach number $\mathrm{M}=u_{r} \sqrt{\frac{\rho_{r}}{p_{r}}}, \operatorname{Re}=\frac{\rho_{r}}{\mu_{r}} u_{r} x_{r}$ is the Reynolds number and $C=$ $\frac{t_{r}^{2} \rho_{r} \gamma_{r}}{x_{r}^{4}}$ the capillarity number.

There are several possibilities to non-dimensionalize the NSK-system. In the sequel we choose $u_{r}=\frac{x_{r}}{t_{r}}$ and consider for a small parameter $\varepsilon>0$ the following scaling:

$$
M=1, \frac{1}{\operatorname{Re}}=\varepsilon^{2}, C=\varepsilon^{2} .
$$

This leads to

$$
\begin{aligned}
\rho_{t^{*}}^{*}+\operatorname{div}^{*}\left(\rho^{*} \mathbf{u}^{*}\right) & =0 \\
\left(\rho^{*} \mathbf{u}^{*}\right)_{t^{*}}+\operatorname{div}^{*}\left(\rho^{*} \mathbf{u}^{*} \otimes \mathbf{u}^{*}\right)+\nabla^{*} p^{*} & =\varepsilon^{2} \operatorname{div}^{*} \boldsymbol{\sigma}_{N S}^{*}+\varepsilon^{2} \gamma^{*} \operatorname{div}^{*} \boldsymbol{\sigma}_{K}^{*}
\end{aligned}
$$

We will call this scaling the capillarity regime. We remark that (4.2),(4.3) is equivalent to

$$
\begin{aligned}
\rho_{t^{*}}^{*}+\operatorname{div}^{*}\left(\rho^{*} \mathbf{u}^{*}\right) & =0 \\
\mathbf{u}_{t^{*}}^{*}+\left(\mathbf{u}^{*} \cdot \nabla^{*}\right) \mathbf{u}^{*}+\nabla^{*} g\left(\rho^{*}\right) & =\varepsilon^{2} \frac{1}{\rho^{*}} \operatorname{div}^{*} \boldsymbol{\sigma}_{N S}^{*}+\varepsilon^{2} \gamma^{*} \nabla^{*} \Delta^{*} \rho^{*} .
\end{aligned}
$$

There are further choices that lead to physically meaningful limits. More details can be found in the introduction.

For simplification we omit the symbol ${ }^{*}$ in the forthcoming considerations. Letting the parameter $\varepsilon \rightarrow 0$ leads to jump conditions of the corresponding sharp interface model, which we will deduce in Section 7. Before that, we need some prerequisites for the asymptotic analysis.

\section{Inner and outer expansions and matching conditions}

We consider the two dimensional case $d=2$. The position of the phase boundary in the sharp interface limit is described by a function $\mathbf{r}(t, s)$, where $s$ is some coordinate parameterizing the interface. We can calculate normal- and tangent vectors as well as the velocity of the interface from r. We want to mention that there is some freedom in the choice of a parameterization, especially we can prescribe any tangential velocity. We will later on see that the tangential velocity is continuous across the interface. Therefore we may choose a particular parameterization such that several terms drop out, cf. Remark 7.2.

The tangent vector pointing in counterclockwise direction is given by

$$
\mathbf{t}(t, s)=\left(\frac{\partial r^{1}}{\partial s}(t, s), \frac{\partial r^{2}}{\partial s}(t, s)\right)^{T}
$$


where $r^{1}$ and $r^{2}$ denote the components of the vector $\mathbf{r}$ in Cartesian coordinates. The inner unit normal to the interface is given by

$$
\boldsymbol{v}(t, s)=\frac{1}{|\mathbf{t}(t, s)|}\left(-\frac{\partial r^{2}}{\partial s}(t, s), \frac{\partial r^{1}}{\partial s}(t, s)\right)^{T} .
$$

The mean curvature, which in two space dimensions coincides with the sum of the principal curvatures, is defined by

$$
\kappa:=\frac{r_{s}^{1} r_{s s}^{2}-r_{s s}^{1} r_{s}^{2}}{\left(\left(r_{s}^{1}\right)^{2}+\left(r_{s}^{2}\right)^{2}\right)^{\frac{3}{2}}}
$$

Next we define the interface velocity and its decomposition into tangential and normal components by

We observe that

$$
\mathbf{w}:=\frac{\partial \mathbf{r}}{\partial t}=w_{t} \mathbf{t}+w_{v} \mathbf{v}
$$

$$
\left(\frac{t^{j}}{|\mathbf{t}|}\right)_{s}=\kappa|\mathbf{t}| v^{j} \quad \text { and } \quad\left(v^{j}\right)_{s}=-t^{j} \kappa, \quad(j=1,2)
$$

and

$$
\left(v^{j}\right)_{\xi} v^{j}=0, \quad\left(\frac{t^{j}}{|\mathbf{t}|}\right)_{\xi} \frac{t^{j}}{|\mathbf{t}|}=0, \quad\left(\frac{t^{j}}{|\mathbf{t}|}\right)_{\xi} v^{j}=-\frac{t^{j}}{|\mathbf{t}|}\left(v^{j}\right)_{\xi},
$$

where the index $\xi$ in the expressions denotes the time or tangential derivative. Note that in equation (5.4) and all subsequent calculations we sum over all indices occurring twice.

For some generic tangent vector $\psi$ the surface divergence in one dimension is defined by

$$
\operatorname{div}_{\Gamma}(\psi)=\frac{1}{|\mathbf{t}|} \frac{\partial(|\mathbf{t}| \psi)}{\partial s}
$$

\section{$5.1 \quad$ Outer setting}

We assume the existence of expansions in $\varepsilon$ for the density and velocity in the bulk phases:

$$
\mathbf{u}\left(t, x^{1}, x^{2} ; \varepsilon\right) \sim \sum_{i=0}^{\infty} \varepsilon^{i} \mathbf{u}_{i}\left(t, x^{1}, x^{2}\right), \quad \rho\left(t, x^{1}, x^{2} ; \varepsilon\right) \sim \sum_{i=0}^{\infty} \varepsilon^{i} \rho_{i}\left(t, x^{1}, x^{2}\right),
$$

where the $\sim$ implicitly means that the infinite sum converges uniformly. Inserting these expansions into the NSK equations leads to the following equations in the first two orders: In the $O\left(\varepsilon^{0}\right)$ order:

$$
\begin{aligned}
\rho_{0, t}+\operatorname{div}\left(\rho_{0} \mathbf{u}_{0}\right) & =0, \\
\left(\mathbf{u}_{0}\right)_{t}+\left(\mathbf{u}_{0} \cdot \nabla\right) \mathbf{u}_{0}+\nabla g\left(\rho_{0}\right) & =0 .
\end{aligned}
$$

In the $\mathcal{O}\left(\varepsilon^{1}\right)$ order:

$$
\begin{aligned}
\rho_{1, t}+\operatorname{div}\left(\rho_{1} \mathbf{u}_{0}+\rho_{0} \mathbf{u}_{1}\right) & =0, \\
\left(\mathbf{u}_{1}\right)_{t}+\left(\mathbf{u}_{0} \cdot \nabla\right) \mathbf{u}_{1}+\left(\mathbf{u}_{1} \cdot \nabla\right) \mathbf{u}_{0}+\nabla\left(g^{\prime}\left(\rho_{0}\right) \rho_{1}\right) & =0 .
\end{aligned}
$$

The conditions at the interface are obtained via matching. 


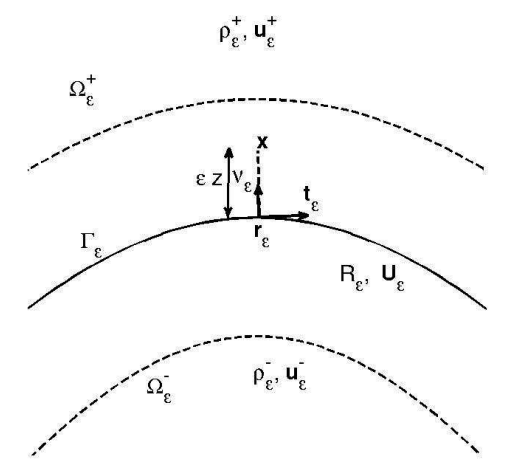

FIG. 2. Boundary layer region

\section{$5.2 \quad$ Inner setting}

Throughout the rest of this paper we will assume that the interface $\Gamma_{\varepsilon}$ defined as

$$
\Gamma_{\varepsilon}(t)=\left\{\left(x^{1}, x^{2}\right) \in \mathbb{R}^{2}: \rho_{\varepsilon}\left(t, x^{1}, x^{2}\right)=\rho_{*}\right\}
$$

is a $C^{1}\left([0, T), C^{2}(\Omega)\right)$-hypersurface. We like to mention that this has not to be the case in general for all times $t$. The value $\rho_{*}$ is some point in the elliptic region, i.e., $p^{\prime}\left(\rho_{*}\right)<0$. The interface $\Gamma_{\varepsilon}$ is explicitly described by $\mathbf{r}_{\varepsilon}$, the inner unit normal $\boldsymbol{v}_{\varepsilon}$ and the tangential vector $\mathbf{t}_{\varepsilon}$ respectively. A point in the neighborhood of the interface is represented by

$$
\left(\begin{array}{l}
x^{1} \\
x^{2}
\end{array}\right)(\tau, s, z)=\mathbf{r}_{\varepsilon}(\tau, s)+\varepsilon z \mathbf{v}_{\varepsilon}(\tau, s),
$$

where $z$ denotes the distance from the interface in normal direction. The reader may note that we could have used $t$ instead of $\tau$ in (5.12), but it will become clear in the subsequent analysis why we want to rename the time variable in inner coordinates. The small parameter $\varepsilon$ is introduced to zoom in the interfacial region. The representation (5.12) can be used to change variables from $\left(x^{1}, x^{2}\right) \leftrightarrow(s, z)$ without changing the time variable.

We suppose a scalar or a Cartesian component of a vector $\psi$ is defined in inner and outer coordinates, i.e., $\psi\left(t, x^{1}, x^{2}\right)=\Psi(\tau, s, z)$. In the following we denote quantities in inner coordinates by capital letters. In particular $R$ is the density in inner coordinates. The partial derivatives of $\psi$ transform as follows:

$$
\left(\begin{array}{l}
\frac{\partial \psi}{\partial x^{1}} \\
\frac{\partial \psi}{\partial x^{2}} \\
\frac{\partial \psi}{\partial t}
\end{array}\right)=\left(\begin{array}{ccc}
(1+\varepsilon z \kappa) \frac{1}{\left|\mathbf{t}_{\varepsilon}\right|^{2}} t_{\varepsilon}^{1} & \varepsilon^{-1} v_{\varepsilon}^{1} & 0 \\
(1+\varepsilon z \kappa) \frac{1}{\left|\mathbf{t}_{\varepsilon}\right|^{2}} t_{\varepsilon}^{2} & \varepsilon^{-1} v_{\varepsilon}^{2} & 0 \\
-(1+\varepsilon z \kappa)\left(\left(w_{t}\right)_{\varepsilon}+\varepsilon z \frac{1}{\left|\mathbf{t}_{\varepsilon}\right|^{2}} t_{\varepsilon}^{i}\left(v_{\varepsilon}^{i}\right)_{\tau}\right) & -\varepsilon^{-1}\left(w_{\nu}\right)_{\varepsilon} & 1
\end{array}\right)\left(\begin{array}{l}
\frac{\partial \Psi}{\partial s} \\
\frac{\partial \Psi}{\partial z} \\
\frac{\partial \Psi}{\partial \tau}
\end{array}\right)+\mathcal{O}\left(\varepsilon^{2}\right),
$$

where $\left(w_{t}\right)_{\varepsilon}=w_{\varepsilon}^{i} \frac{t_{\varepsilon}^{i}}{\left|\mathbf{t}_{\varepsilon}\right|^{2}}$ and $\left(w_{\nu}\right)_{\varepsilon}=w_{\varepsilon}^{i} v_{\varepsilon}^{i}$, for any $(\tau, s, z) \in[0, T) \times I \times \mathbb{R}$, where $I \subset \mathbb{R}$ is some compact interval used to parameterize the interface. 
Accordingly to the outer expansion (5.6) we assume the existence of inner expansions in $\varepsilon$. In particular we assume

$$
\mathbf{U}(\tau, s, z ; \varepsilon) \sim \sum_{i=0}^{\infty} \varepsilon^{i} \mathbf{U}_{i}(\tau, s, z), \quad R(\tau, s, z ; \varepsilon) \sim \sum_{i=0}^{\infty} \varepsilon^{i} R_{i}(\tau, s, z),
$$

where $\mathbf{U}_{i}, R_{i}$ do not depend on $\varepsilon$ for all $i \in \mathbb{N}_{0}$. Furthermore we suppose expansions of the geometric quantity

$$
\mathbf{r}_{\varepsilon}(\tau, s) \sim \sum_{i=0}^{\infty} \varepsilon^{i} \mathbf{r}_{i}(\tau, s),
$$

which induces $\varepsilon$-expansions of $\boldsymbol{v}_{\varepsilon}, \mathbf{t}_{\varepsilon}$ and $\mathbf{w}_{\varepsilon}$.

Particularly we get the following expansions, up to terms of order $\odot\left(\varepsilon^{2}\right)$ :

$$
\begin{aligned}
\left(w_{\nu}\right)_{\varepsilon}= & \underbrace{v_{0}^{i} w_{0}^{i}}_{=: w_{\nu 0}}+\varepsilon \underbrace{\left(v_{1}^{i} w_{0}^{i}+v_{0}^{i} w_{1}^{i}\right)}_{=: w_{\nu 1}}+\mathcal{O}\left(\varepsilon^{2}\right), \\
\left(U_{\nu}\right)_{\varepsilon}= & \underbrace{v_{0}^{i} U_{0}^{i}}_{=: U_{\nu 0}}+\varepsilon \underbrace{\left(v_{1}^{i} U_{0}^{i}+v_{0}^{i} U_{1}^{i}\right)}_{=: U_{\nu 1}}+\mathcal{O}\left(\varepsilon^{2}\right), \\
\left(U_{t}\right)_{\varepsilon}= & \underbrace{\frac{t_{0}^{i} U_{0}^{i}}{\left|\mathbf{t}_{0}\right|^{2}}}_{=: U_{t 0}}+\underbrace{\left(\frac{t_{1}^{i} U_{0}^{i}}{\left|\mathbf{t}_{0}\right|^{2}}-2 \frac{t_{0}^{i} U_{0}^{i} t_{0}^{j} t_{1}^{j}}{\left|\mathbf{t}_{0}\right|^{4}}+\frac{t_{0}^{i} U_{1}^{i}}{\left|\mathbf{t}_{0}\right|^{2}}\right)}_{=: U_{t 1}}+\mathcal{O}\left(\varepsilon^{2}\right), \\
\left(w_{t}\right)_{\varepsilon}= & \underbrace{\frac{t_{0}^{i} w_{0}^{i}}{\left|\mathbf{t}_{0}\right|^{2}}}_{=: w_{t 0}}+\varepsilon \underbrace{\left(\frac{t_{1}^{i} w_{0}^{i}}{\left|\mathbf{t}_{0}\right|^{2}}-2 \frac{t_{0}^{i} w_{0}^{i} t_{0}^{j} t_{1}^{j}}{\left|\mathbf{t}_{0}\right|^{4}}+\frac{t_{0}^{i} w_{1}^{i}}{\left|\mathbf{t}_{0}\right|^{2}}\right)}_{=: w_{t 1}}+\mathcal{O}\left(\varepsilon^{2}\right), \\
J_{\varepsilon}:= & R_{\varepsilon}\left(\left(U_{\nu}\right)_{\varepsilon}-\left(w_{\nu}\right)_{\varepsilon}\right) \\
= & \underbrace{R_{0}\left(U_{\nu 0}-w_{\nu 0}\right)}_{=: J_{0}}+\varepsilon \underbrace{\left(R_{1}\left(U_{\nu 0}-w_{\nu 0}\right)+R_{0}\left(U_{\nu 1}-w_{\nu 1}\right)\right)}_{=: J_{1}}+\mathcal{O}\left(\varepsilon^{2}\right) .
\end{aligned}
$$

With respect to the expansions of the geometric quantities we observe

$$
1=\left|\boldsymbol{v}_{\varepsilon}\right|^{2}=v_{0}^{i} v_{0}^{i}+2 \varepsilon v_{0}^{i} v_{1}^{i}+\mathcal{O}\left(\varepsilon^{2}\right),
$$

which implies

Furthermore we have

$$
v_{0}^{i} v_{0}^{i}=1, \quad v_{0}^{i} v_{1}^{i}=0
$$

$$
0=t_{\varepsilon}^{i} v_{\varepsilon}^{i}=t_{0}^{i} v_{0}^{i}+\varepsilon\left(t_{1}^{i} v_{0}^{i}+t_{0}^{i} v_{1}^{i}\right)+\mathcal{O}\left(\varepsilon^{2}\right),
$$

which implies

$$
t_{0}^{i} v_{0}^{i}=0, \quad v_{0}^{i} t_{1}^{i}+v_{1}^{i} t_{0}^{i}=0 .
$$

Additionally an easy calculation shows

$$
\left(v_{0}^{i}\right)_{s}=-\kappa_{0} t_{0}^{i}, \quad\left(\frac{t_{0}^{i}}{\left|\mathbf{t}_{0}\right|}\right)_{s}=\kappa_{0}\left|\mathbf{t}_{0}\right| v_{0}^{i}
$$

Using (5.12), (5.14) and (5.15) we get the inner equations by comparing coefficients of different powers of $\varepsilon$. 


\subsection{Matching conditions}

Inner- and outer quantities are matched by the usual procedure, see [7] for details. For convenience of the reader we sketch the arguments. Let $\psi$ and $\Psi$ be any function possessing expansions like (5.6) and (5.14). Near the interfacial layer we formally equate the two expansions

$$
\Psi(\tau, s, z ; \varepsilon) \sim \sum_{i=0}^{\infty} \varepsilon^{i} \Psi_{i}(\tau, s, z)=\sum_{i=0}^{\infty} \varepsilon^{i} \psi_{i}\left(t, \mathbf{r}_{\varepsilon}(\tau, s)+\varepsilon z \boldsymbol{v}_{\varepsilon}(\tau, s)\right) \sim \psi\left(t, x^{1}, x^{2} ; \varepsilon\right)
$$

Then we expand the right-hand side in a Taylor series yielding

$$
\sum_{i=0}^{\infty} \varepsilon^{i} \Psi_{i}(\tau, s, z)=\sum_{i=0}^{N} \varepsilon^{i} P_{i}^{ \pm}(\tau, s, z)+\varepsilon^{N+1} R_{N+1}^{ \pm},
$$

where

$$
P_{i}^{ \pm}=\left.\frac{1}{i !} \frac{d^{i}}{d \varepsilon^{i}} \psi\left(\tau, \mathbf{r}_{\varepsilon}(\tau, s)+\varepsilon z \boldsymbol{v}_{\varepsilon}(\tau, s)\right)\right|_{\varepsilon=0}
$$

and $R_{N+1}^{ \pm}$is a Lagrange remainder. We observe that $P_{i}^{ \pm}$is a polynomial of order $i$ in $z$ with coefficients depending on $\mathbf{r}$ and its derivatives and on $\left(x^{1}, x^{2}\right)$-derivatives of $\psi$. We will assume that all the $\left(x^{1}, x^{2}\right)$-derivatives of $\psi$ up to order $N+1$ are bounded in a neighborhood of the interface. The polynomials $P_{i}^{+}, P_{i}^{-}$are valid for $z>0$ and $z<0$ respectively. Now let $z \rightarrow \pm \infty$ with $\varepsilon$ coupled to $z$ such that $\varepsilon z^{N+1} \rightarrow 0$ but otherwise arbitrary. Then the remainder term in (5.26) has higher order than all the preceding terms. This fact leads to the identification of the inner quantities with the Taylor polynomials for $z \rightarrow \infty$, i.e.,

$$
\Psi_{i}(\tau, s, z)=P_{i}^{ \pm}(\tau, s, z)+o(1) \text { for } z \rightarrow \pm \infty .
$$

Applying this to the first two orders of the asymptotic expansions shows

$$
\begin{array}{lr}
\Psi_{0}(\tau, s, z) \rightarrow \psi_{0}^{ \pm}\left(\tau, \mathbf{r}_{0}(\tau, s)\right) & z \rightarrow \pm \infty, \\
\Psi_{1}(\tau, s, z)-\psi_{1}^{ \pm}\left(\tau, \mathbf{r}_{0}(\tau, s)\right)-\left(\frac{\partial \psi_{0}}{\partial x^{j}}\right)^{ \pm}\left(\tau, \mathbf{r}_{0}(\tau, s)\right)\left(r_{1}^{j}(\tau, s)=v_{0}^{j}(\tau, s) z\right)+o(1) & z \rightarrow \pm \infty .
\end{array}
$$

By differentiating both sides in (5.25) with respect to $s, z$ or $\tau$ we can derive further matching conditions:

$$
\begin{array}{lcc}
\Psi_{0, \tau} \rightarrow & \left(\frac{\partial \psi_{0}}{\partial x^{j}}\right)^{ \pm}\left(\tau, \mathbf{r}_{0}(\tau, s)\right) w_{0}^{j}(\tau, s)+\left(\frac{\partial \psi_{0}}{\partial t}\right)^{ \pm}\left(\tau, \mathbf{r}_{0}(\tau, s)\right) & z \rightarrow \pm \infty \\
\Psi_{0, s} \rightarrow & \left(\frac{\partial \psi_{0}}{\partial x^{j}}\right)^{ \pm}\left(\tau, \mathbf{r}_{0}(\tau, s)\right) t_{0}^{j}(\tau, s) & z \rightarrow \pm \infty \\
\Psi_{0, z} \rightarrow & 0 & z \rightarrow \pm \infty \\
\Psi_{1, z} \rightarrow & \left(\frac{\partial \psi_{0}}{\partial x^{j}}\right)^{ \pm}\left(\tau, \mathbf{r}_{0}(\tau, s)\right) v_{0}^{j}(\tau, s) & z \rightarrow \pm \infty
\end{array}
$$


We will assume that all these limits are attained superlinearly fast. When we redefine

$$
\begin{aligned}
& \psi_{0}^{+}(s, \tau):=\lim _{z>0, z \rightarrow 0} \psi_{0}\left(\tau, \mathbf{r}_{0}(s, \tau)+z \boldsymbol{v}_{0}(s, \tau)\right), \\
& \psi_{0}^{-}(s, \tau):=\lim _{z<0, z \rightarrow 0} \psi_{0}\left(\tau, \mathbf{r}_{0}(s, \tau)+z \boldsymbol{v}_{0}(s, \tau)\right),
\end{aligned}
$$

we can rewrite (5.31) and (5.32) as

$$
\begin{aligned}
& \Psi_{0, \tau} \rightarrow\left(\psi_{0}^{ \pm}\right)_{\tau} \quad z \rightarrow \pm \infty \\
& \Psi_{0, s} \rightarrow\left(\psi_{0}^{ \pm}\right)_{s} \quad z \rightarrow \pm \infty
\end{aligned}
$$

\section{Main results}

In our considerations we assume that for all relevant quantities there exists an asymptotic expansion in $\varepsilon$. In particular we consider solutions of the structure given below:

Definition 6.1 Let $\left(\rho_{\varepsilon}, \mathbf{u}_{\varepsilon}\right)$ be a classical solution of (4.4), (4.5) existing in the time interval [0,T) with inner and outer expansions as in (5.6) and (5.14).

In addition let

$$
\begin{aligned}
\Omega_{\varepsilon}^{-}(t) & :=\left\{\left(x^{1}, x^{2}\right) \in \mathbb{R}^{2}: \rho_{\varepsilon}\left(t, x^{1}, x^{2}\right)<\rho_{*}\right\}, \\
\Omega_{\varepsilon}^{+}(t) & :=\left\{\left(x^{1}, x^{2}\right) \in \mathbb{R}^{2}: \rho_{\varepsilon}\left(t, x^{1}, x^{2}\right)>\rho_{*}\right\}, \\
\Gamma_{\varepsilon}(t) & :=\left\{\left(x^{1}, x^{2}\right) \in \mathbb{R}^{2}: \rho_{\varepsilon}\left(t, x^{1}, x^{2}\right)=\rho_{*}\right\},
\end{aligned}
$$

for $t \in[0, T)$ and let $\Gamma_{\varepsilon}$ be a $C^{1}\left([0, T), C^{2}\left(\mathbb{R}^{2}\right)\right)$-hypersurface with an asymptotic $\varepsilon$-expansion as in $(5.15)$.

We call $\left(\rho_{0}, \mathbf{u}_{0}, \rho_{1}, \mathbf{u}_{1}\right)$ an outer solution if

$$
\begin{aligned}
& \rho_{0}, \rho_{1} \in C^{1}\left([0, T), C^{0}\left(\bar{\Omega}_{0}^{ \pm}(t)\right)\right) \cap C^{0}\left([0, T), C^{1}\left(\bar{\Omega}_{0}^{ \pm}(t)\right)\right), \\
& \mathbf{u}_{0}, \mathbf{u}_{1} \in C^{1}\left([0, T), C^{0}\left(\bar{\Omega}_{0}^{ \pm}(t), \mathbb{R}^{2}\right)\right) \cap C^{0}\left([0, T), C^{1}\left(\bar{\Omega}_{0}^{ \pm}(t), \mathbb{R}^{2}\right)\right)
\end{aligned}
$$

and $\left(\rho_{0}, \mathbf{u}_{0}, \rho_{1}, \mathbf{u}_{1}\right)$ satisfies

$$
\begin{aligned}
\rho_{0, t}+\operatorname{div}\left(\rho_{0} \mathbf{u}_{0}\right) & =0, \\
\left(\mathbf{u}_{0}\right)_{t}+\left(\mathbf{u}_{0} \cdot \nabla\right) \mathbf{u}_{0}+\nabla g\left(\rho_{0}\right) & =0, \\
\rho_{1, t}+\operatorname{div}\left(\rho_{1} \mathbf{u}_{0}+\rho_{0} \mathbf{u}_{1}\right) & =0, \\
\left(\mathbf{u}_{1}\right)_{t}+\left(\mathbf{u}_{0} \cdot \nabla\right) \mathbf{u}_{1}+\left(\mathbf{u}_{1} \cdot \nabla\right) \mathbf{u}_{0}+\nabla\left(g^{\prime}\left(\rho_{0}\right) \rho_{1}\right) & =0 .
\end{aligned}
$$

We call $\left(R_{0}, \mathbf{U}_{0}, R_{1}, \mathbf{U}_{1}\right)$ an inner solution if

$$
\begin{aligned}
& R_{0}, R_{1} \in C^{1}\left([0, T) \times I, C^{3}(\mathbb{R})\right), \\
& \mathbf{U}_{0}, \mathbf{U}_{1} \in C^{1}\left([0, T) \times I, C^{2}\left(\mathbb{R}, \mathbb{R}^{2}\right)\right),
\end{aligned}
$$

where $I$ is the compact interval used to parameterize the interface and $\left(R_{0}, \mathbf{U}_{0}, R_{1}, \mathbf{U}_{1}\right)$ satisfies

$$
-w_{\nu 0} R_{0, z}+\left(U_{\nu 0} R_{0}\right)_{z}=0
$$




$$
\begin{array}{r}
-w_{\nu 0}\left(U_{0}^{j}\right)_{z}+U_{\nu 0}\left(U_{0}^{j}\right)_{z}+v_{0}^{j} g\left(R_{0}\right)_{z}-v_{0}^{j} \gamma R_{0, z z z}=0, \\
-w_{\nu 1} R_{0, z}-w_{\nu 0} R_{1, z}-w_{t 0} R_{0, s}+R_{0, \tau}+\frac{t_{0}^{i}}{\left|\mathbf{t}_{0}\right|^{2}}\left(R_{0} U_{0}^{i}\right)_{s}+\left(R_{1} U_{\nu 0}+R_{0} U_{\nu 1}\right)_{z}=0, \\
-w_{\nu 1}\left(U_{0}^{j}\right)_{z}-w_{\nu 0}\left(U_{1}^{j}\right)_{z}+\left(U_{0}^{j}\right)_{\tau}-w_{t 0}\left(U_{0}^{j}\right)_{s}+U_{\nu 1}\left(U_{0}^{j}\right)_{z}+U_{\nu 0}\left(U_{1}^{j}\right)_{z} \\
+U_{t 0}\left(U_{0}^{j}\right)_{s}+g\left(R_{0}\right)_{z} v_{1}^{j}+\left(g^{\prime}\left(R_{0}\right) R_{1}\right)_{z} v_{0}^{j}+g\left(R_{0}\right)_{s} \frac{t_{0}^{j}}{\left|\mathbf{t}_{0}\right|^{2}} \\
-(\lambda+2 \mu) v_{0}^{j} \frac{1}{R_{0}}\left(U_{\nu 0}\right)_{z z} \\
=\mu t_{0}^{j} \frac{1}{R_{0}}\left(U_{t 0}\right)_{z z}+\gamma v_{0}^{j} R_{1, z z z}+\gamma v_{1}^{j} R_{0, z z z}-\gamma \kappa_{0} v_{0}^{j} R_{0, z z}+\gamma \frac{t_{0}^{j}}{\left|\mathbf{t}_{0}\right|^{2}} R_{0, s z z} .
\end{array}
$$

We call $\left(\rho_{0}, \mathbf{u}_{0}, \rho_{1}, \mathbf{u}_{1}, R_{0}, \mathbf{U}_{0}, R_{1}, \mathbf{U}_{1}\right)$ a matching solution, when $\left(\rho_{0}, \mathbf{u}_{0}, \rho_{1}, \mathbf{u}_{1}\right)$ is an outer solution, $\left(R_{0}, \mathbf{U}_{0}, R_{1}, \mathbf{U}_{1}\right)$ is an inner solution and both are linked by the matching conditions (5.29)-(5.34). We call a matching solution admissible, if the mass flux $\rho_{0}\left(u_{\nu 0}-w_{\nu 0}\right) \neq 0$.

REMARK 6.2 Equations (6.3)-(6.6) were motivated in Section 5.1. To derive (6.9)-(6.12) we perform the coordinate change (5.12) in (4.4) and (4.5), which changes the partial derivatives according to (5.13). Then we insert the expansions (5.14) and compare coefficients of different powers of $\varepsilon$.

REMARK 6.3 Real phase transitions take only place if the mass flux is non-zero, i.e., $\rho_{0}\left(u_{v 0}-\right.$ $\left.w_{\nu 0}\right) \neq 0$. Therefore, we have chosen this condition as an admissibility condition. We like to point out that the subsequent analysis does not cover the case $\rho_{0}\left(u_{\nu 0}-w_{\nu 0}\right)=0$.

For every quantity $\psi$ in outer variables having an asymptotic expansion in $\varepsilon$ we will use the following notation for the jump of its leading orders at the interface:

$$
\llbracket \psi \rrbracket_{0,1}:=\llbracket \psi_{0} \rrbracket+\varepsilon \llbracket \psi_{1} \rrbracket \quad \text { and } \quad\{\psi\}_{0,1}:=\left\{\psi_{0}\right\}+\varepsilon\left\{\psi_{1}\right\} .
$$

Now we are well-prepared to state our main results.

THEOREM 6.4 If $\left(\rho_{0}, \mathbf{u}_{0}, \rho_{1}, \mathbf{u}_{1}, R_{0}, \mathbf{U}_{0}, R_{1}, \mathbf{U}_{1}\right)$ is an admissible matching solution defined as in Definition 6.1, then the following jump conditions are fulfilled:

$$
\begin{aligned}
\llbracket \rho_{\varepsilon}\left(\left(u_{v}\right)_{\varepsilon}-\left(w_{\nu}\right)_{\varepsilon}\right) \rrbracket_{0,1} & =-\varepsilon \frac{\partial \rho_{\Gamma}}{\partial \tau}-\varepsilon\left(\operatorname{div}_{\Gamma}\left(w_{t 0}\right)-\kappa_{0} w_{\nu 0}\right) \rho_{\Gamma}, \\
\llbracket \rho_{\varepsilon}\left(\left(u_{v}\right)_{\varepsilon}-\left(w_{v}\right)_{\varepsilon}\right)\left(\mathbf{u}_{\varepsilon}-\mathbf{w}_{\varepsilon}\right)+p\left(\rho_{\varepsilon}\right) \boldsymbol{v}_{\varepsilon} \rrbracket_{0,1} & =-\varepsilon \rho_{\Gamma} \frac{\partial \mathbf{w}_{0}}{\partial \tau}+\varepsilon \operatorname{div}_{\Gamma}\left(\sigma_{\Gamma}\right), \\
\llbracket \frac{1}{2}\left(\frac{j_{\varepsilon}}{\rho_{\varepsilon}}\right)^{2}+g\left(\rho_{\varepsilon}\right) \prod_{0,1} & =-\varepsilon \int_{0}^{\infty} v_{0}^{j}\left(U_{0}^{j}-\left(u_{0}^{j}\right)^{+}\right)_{\tau} d z \\
& -\varepsilon \int_{-\infty}^{0} v_{0}^{j}\left(U_{0}^{j}-\left(u_{0}^{j}\right)^{-}\right)_{\tau} d z \\
& -\varepsilon(\lambda+2 \mu) j_{0} \int_{-\infty}^{\infty}\left(\left(\frac{1}{R_{0}}\right)_{z}\right)^{2} d z,
\end{aligned}
$$


where

$$
j_{\varepsilon}:=\rho_{\varepsilon}\left(\left(u_{\nu}\right)_{\varepsilon}-\left(w_{v}\right)_{\varepsilon}\right)
$$

is the massflux across the interface and

$$
\begin{aligned}
\rho_{\Gamma} & :=\int_{0}^{\infty}\left(R_{0}-\rho_{0}^{+}\right) d z-\int_{-\infty}^{0}\left(R_{0}-\rho_{0}^{-}\right) d z \\
\sigma_{\Gamma} & :=\sigma_{\Gamma}^{j}=\left(\int_{0}^{\infty}\left(\frac{j_{0}^{2}}{R_{0}}-\frac{j_{0}^{2}}{\rho_{0}^{+}}+\gamma R_{0, z}^{2}\right) d z+\int_{-\infty}^{0} \ldots d z\right) \frac{t_{0}^{j}}{\left|\mathbf{t}_{0}\right|^{2}} .
\end{aligned}
$$

REMARK 6.5 We want to emphasize that (6.13) and (6.14) recover (3.3) and (3.4) respectively and we will show in Theorem 6.7 that (6.13)-(6.15) are in agreement with (3.10).

REMARK 6.6 The zeroth orders of (6.13)-(6.15) imply that the states $\rho_{0}^{ \pm}$are the Maxwell points of the free energy density function

$$
W_{j_{0}}(\rho):=W(\rho)-\frac{j_{0}^{2}}{2 \rho} .
$$

The Maxwell points $\alpha$ and $\beta$ are the uniquely determined points satisfying

$$
W_{j_{0}}^{\prime}(\alpha)=W_{j_{0}}^{\prime}(\beta)=\frac{W_{j_{0}}(\beta)-W_{j_{0}}(\alpha)}{\beta-\alpha} .
$$

THEOREM 6.7 If $\left(\rho_{0}, \mathbf{u}_{0}, \rho_{1}, \mathbf{u}_{1}, R_{0}, \mathbf{U}_{0}, R_{1}, \mathbf{U}_{1}\right)$ is an admissible matching solution defined as in Definition 6.1, then for $\varepsilon$ sufficiently small the jump conditions derived in Theorem 6.4 imply the inequality

$$
\begin{aligned}
0 \geqslant- & \varepsilon(\lambda+2 \mu) j_{0}^{2} \int_{-\infty}^{\infty}\left(\left(\frac{1}{R_{0}}\right)_{z}\right)^{2} d z \\
= & \varepsilon \frac{\partial W_{\Gamma}}{\partial \tau}-\varepsilon\left(\gamma_{\Gamma}-W_{\Gamma}\right)\left(\operatorname{div}_{\Gamma}\left(\left(w_{t}\right)_{\varepsilon}\right)-\kappa_{\varepsilon}\left(w_{\nu}\right)_{\varepsilon}\right)+\llbracket j_{\varepsilon} \rrbracket_{0,1}\left\{W^{\prime}\left(\rho_{\varepsilon}\right)+\frac{\left|\mathbf{u}_{\varepsilon}-\mathbf{w}_{\varepsilon}\right|^{2}}{2}\right\}_{0,1} \\
& +\left\{j_{\varepsilon}\right\}_{0,1} \llbracket \llbracket W^{\prime}\left(\rho_{\varepsilon}\right)+\frac{\left|\mathbf{u}_{\varepsilon}-\mathbf{w}_{\varepsilon}\right|^{2}}{2} \rrbracket_{0,1} .
\end{aligned}
$$

Recall that the surface tension $\gamma_{\Gamma}$ is related to the surface stress tensor by (3.8). The interface mass density $\rho_{\Gamma}$ and the surface stress tensor are as in (6.17), (6.18) and the Helmholtz surface energy density $W_{\Gamma}$ is given by

$$
\begin{aligned}
W_{\Gamma}=\int_{0}^{\infty}\left(W\left(R_{0}\right)-W\left(\rho_{0}^{+}\right)\right. & \left.+\frac{1}{2} \frac{j_{0}^{2}}{R_{0}}-\frac{1}{2} \frac{j_{0}^{2}}{\rho_{0}^{+}}+\frac{\gamma}{2} R_{0, z}^{2}\right) d z \\
& +\int_{-\infty}^{0}\left(W\left(R_{0}\right)-W\left(\rho_{0}^{-}\right)+\frac{1}{2} \frac{j_{0}^{2}}{R_{0}}-\frac{1}{2} \frac{j_{0}^{2}}{\rho_{0}^{-}}+\frac{\gamma}{2} R_{0, z}^{2}\right) d z
\end{aligned}
$$

The inequality (6.19) is identical to the entropy inequality (3.10). Note that in (6.19) the interface terms contribute in the first order of $\varepsilon$. Thus the jump conditions derived in Theorem 6.4 are compatible to the second law of thermodynamics. 
REMARK 6.8 A straightforward computation shows

$$
W_{\Gamma}-\gamma_{\Gamma}=\rho_{\Gamma}\left(g\left(\rho_{0}^{ \pm}\right)+\frac{1}{2}\left(\frac{j_{0}}{\rho_{0}^{ \pm}}\right)^{2}\right)
$$

which is a special case of the Gibbs adsorption law, see [12].

REMARK 6.9 The inequality (6.19) can be obtained by straightforward but cumbersome calculations using asymptotic analysis to the entropy inequality in the bulk (see (2.6)), which is derived in [9].

\section{Proof of Theorem 6.4}

\section{$7.1 \mathcal{O}\left(\varepsilon^{-1}\right)$-terms}

In the following lemma we will establish the zeroth order of (6.13), (6.14) and (6.15).

LEMMA 7.1 If $\left(\rho_{0}, \mathbf{u}_{0}, \rho_{1}, \mathbf{u}_{1}, R_{0}, \mathbf{U}_{0}, R_{1}, \mathbf{U}_{1}\right)$ is an admissible matching solution defined as in Definition 6.1, then the following jump conditions of zeroth order are satisfied:

$$
\begin{aligned}
\llbracket \rho_{\varepsilon}\left(\left(u_{v}\right)_{\varepsilon}-\left(w_{v}\right)_{\varepsilon}\right) \rrbracket_{0} & =0, \\
\llbracket \rho_{\varepsilon}\left(\left(u_{v}\right)_{\varepsilon}-\left(w_{v}\right)_{\varepsilon}\right)\left(\mathbf{u}_{\varepsilon}-\mathbf{w}_{\varepsilon}\right)+p\left(\rho_{\varepsilon}\right) \boldsymbol{v}_{\varepsilon} \rrbracket_{0} & =0, \\
\llbracket\left(u_{t}\right)_{\varepsilon} \rrbracket_{0} & =0, \\
\llbracket \frac{1}{2}\left(\frac{j_{\varepsilon}}{\rho_{\varepsilon}}\right)^{2}+g\left(\rho_{\varepsilon}\right) \rrbracket_{0} & =0 .
\end{aligned}
$$

Proof. We recall that the mass flux across the interface is given by $j=\rho\left(u_{v}-w_{v}\right)$. Hence equation (6.9) implies $J_{0, z}=0$ and therefore we get the jump condition (7.1), i.e.,

$$
\llbracket j \rrbracket_{0}=0 .
$$

Multiplying (6.10) with the zeroth order of the tangent vector $\mathbf{t}_{0}$ we obtain

$$
\frac{J_{0}}{R_{0}}\left(U_{t 0}\right)_{z}=0
$$

Hence, the leading order of the tangent velocity $U_{t 0}$ is constant across the interface. Furthermore to solve (6.9) and (6.10) it is sufficient to substitute $U_{\nu 0}=\frac{J_{0}}{R_{0}}-w_{\nu 0}$ in (6.10) and to solve (6.10) times $\boldsymbol{v}_{0}$ which gives

$$
\frac{J_{0}}{R_{0}}\left(\frac{J_{0}}{R_{0}}\right)_{z}+g\left(R_{0}\right)_{z}=\gamma R_{0, z z z} .
$$

By Proposition 1.2 in the work of Benzoni-Gavage et. al. [4] there exist values $\rho_{0}^{ \pm}$satisfying certain jump conditions which we will state in (7.9) and (7.12) below. Given these states $\rho_{0}^{ \pm}$there exists a solution $R_{0}$ of (7.7) attaining $\rho_{0}^{ \pm}$as boundary values.

In the sequel we will show that (7.9) and (7.12) are necessary conditions for boundary values of solutions of (7.7). From (7.7) we infer

$$
\frac{1}{2}\left(\frac{J_{0}}{R_{0}}\right)^{2}+g\left(R_{0}\right)=\gamma R_{0, z z}+c_{1}
$$


for some $c_{1} \in \mathbb{R}$. Using the matching conditions (5.29) and (5.33) this implies

$$
c_{1}=\frac{1}{2}\left(\frac{j_{0}}{\rho_{0}^{-}}\right)^{2}+g\left(\rho_{0}^{-}\right)=\frac{1}{2}\left(\frac{j_{0}}{\rho_{0}^{+}}\right)^{2}+g\left(\rho_{0}^{+}\right) .
$$

Multiplying (7.7) by $R_{0}$ we get

$$
J_{0}\left(\frac{J_{0}}{R_{0}}\right)_{z}+p\left(R_{0}\right)_{z}=\gamma R_{0} R_{0, z z z},
$$

which implies

$$
\frac{J_{0}^{2}}{R_{0}}+p\left(R_{0}\right)=\gamma\left(R_{0} R_{0, z z}-\frac{1}{2}\left(R_{0, z}\right)^{2}\right)+c_{2}
$$

for some $c_{2}$ in $\mathbb{R}$. Applying (5.29) and (5.33) to (7.11) we find

$$
c_{2}=\frac{j_{0}^{2}}{\rho_{0}^{-}}+p\left(\rho_{0}^{-}\right)=\frac{j_{0}^{2}}{\rho_{0}^{+}}+p\left(\rho_{0}^{+}\right) .
$$

REMARK 7.2 In the proof of Lemma 7.1 we have seen that the tangential velocity is constant across the interface and up to now we have not chosen a specific parameterization of the interface. So we are free to impose

$$
U_{0}^{i}=w_{t 0} t_{0}^{i}+U_{\nu 0} v_{0}^{i}, \quad \text { i.e., } U_{t 0}=w_{t 0} .
$$

We want to point out that the admissibility condition from Definition 6.1 is crucial to establish the equality of the tangent velocities of the fluid and the interface.

\subsection{Prerequisites for $\bigcirc(1)$-terms}

The following technical lemmata are used to derive the first order jump conditions. Lemma 7.4 shows solvability criteria for the inner equations on an abstract level and is exploited in Lemma 7.5.

Lemma 7.3 Let $Q \in C^{2}(\mathbb{R})$ be given with

$$
Q_{z}(z), Q_{z z}(z) \rightarrow 0, \quad \text { for }|z| \rightarrow \infty .
$$

Further, define

$$
\varphi(z)=\int_{0}^{z} \frac{1}{\left(Q_{z}(\tilde{z})\right)^{2}} d \tilde{z}
$$

Then

$$
\left|\varphi(z) Q_{z}(z)\right| \rightarrow \infty, \quad \text { for }|z| \rightarrow \infty .
$$

Proof. Due to (7.14) there exists some $K>0$ such that

$$
\left|Q_{z}(z)\right|<\varepsilon,\left|Q_{z z}(z)\right|<\varepsilon, \quad \text { for }|z|>K .
$$


We consider some pair $(z, \bar{z})$ with $|\bar{z}|>K+1,|z|>K$ and $z \bar{z}>0$. Then we have

$$
\left|Q_{z}(z)-Q_{z}(\bar{z})\right|=\left|\int_{\bar{z}}^{z} Q_{z z}(\tilde{z}) d \tilde{z}\right| \leqslant|z-\bar{z}| \varepsilon
$$

Therefore

$$
\left|Q_{z}(z)\right| \leqslant 2\left|Q_{z}(\bar{z})\right|, \quad \text { for all }|z-\bar{z}| \leqslant \frac{\left|Q_{z}(\bar{z})\right|}{\varepsilon}(<1) .
$$

Next we define $z^{*}:=\bar{z}-\operatorname{sgn}(\bar{z}) \frac{\left|Q_{z}(\bar{z})\right|}{\varepsilon}$ and find

$$
|\varphi(\bar{z})| \geqslant\left|\int_{z^{*}}^{\bar{z}}\left(\frac{1}{Q_{z}(\tilde{z})}\right)^{2} d \tilde{z}\right| \geqslant\left|\bar{z}-z^{*}\right| \frac{1}{4}\left|\frac{1}{Q_{z}(\bar{z})}\right|^{2},
$$

which implies

$$
\left|\varphi(\bar{z}) Q_{z}(\bar{z})\right| \geqslant \frac{1}{4}\left|\bar{z}-z^{*}\right|\left|\frac{1}{Q_{z}(\bar{z})}\right|=\frac{1}{4 \varepsilon} .
$$

Hence for every $\varepsilon>0$ we find some $K>0$ such that

$$
\left|\varphi(\bar{z}) Q_{z}(\bar{z})\right| \geqslant \frac{1}{4 \varepsilon} \quad \text { for all }|\bar{z}|>K+1,
$$

which proves (7.15).

LEMMA 7.4 Let $\left(\rho_{0}, \mathbf{u}_{0}, \rho_{1}, \mathbf{u}_{1}, R_{0}, \mathbf{U}_{0}, R_{1}, \mathbf{U}_{1}\right)$ be an admissible matching solution and let the operator

$$
L: W^{3,1}(\mathbb{R}) \times W^{1,1}(\mathbb{R}) \times W^{1,1}(\mathbb{R}) \rightarrow L^{1}(\mathbb{R}) \times L^{1}(\mathbb{R}) \times L^{1}(\mathbb{R})
$$

be given by

$$
L\left(\begin{array}{c}
A \\
B \\
C
\end{array}\right):=\left(\begin{array}{c}
\left(A\left(U_{\nu 0}-w_{\nu 0}\right)+R_{0} B\right)_{z} \\
\left(\left(U_{\nu 0}-w_{\nu 0}\right) B+g^{\prime}\left(R_{0}\right) A-\gamma A_{z z}\right)_{z} \\
\left(U_{\nu 0}-w_{\nu 0}\right) C_{z}
\end{array}\right)
$$

Then

$$
L\left(\begin{array}{c}
A \\
B \\
C
\end{array}\right)=\left(\begin{array}{c}
f_{1} \\
f_{2} \\
f_{3}
\end{array}\right), \quad f_{1}, f_{2}, f_{3} \in L^{1}(\mathbb{R}),
$$

has a solution if and only if

$$
\begin{aligned}
& \int_{-\infty}^{\infty} f_{1} d z=0 \\
& \int_{-\infty}^{\infty} f_{2} d z=0 \\
& \int_{-\infty}^{\infty} R_{0} f_{3} d z=0 \\
& \int_{-\infty}^{\infty} \frac{j_{0}}{R_{0}} f_{1}+R_{0} f_{2} d z=0
\end{aligned}
$$


Proof. By the Fredholm alternative theorem the system (7.21) is solvable if and only if the right hand side satisfies

$$
\int_{-\infty}^{\infty} f_{1} \cdot \tilde{A}+f_{2} \cdot \tilde{B}+f_{3} \cdot \tilde{C} d z=0
$$

for every solution $(\tilde{A}, \tilde{B}, \tilde{C})^{T}$ of the homogeneous problem for the adjoint operator. The adjoint operator

$$
L^{*}: L^{\infty}(\mathbb{R}) \times L^{\infty}(\mathbb{R}) \times L^{\infty}(\mathbb{R}) \rightarrow W^{-3,1}(\mathbb{R}) \times W^{-1,1}(\mathbb{R}) \times W^{-1,1}(\mathbb{R})
$$

is given by

$$
L^{*}\left(\begin{array}{c}
\tilde{A} \\
\tilde{B} \\
\tilde{C}
\end{array}\right):=\left(\begin{array}{c}
-\left(U_{\nu 0}-w_{\nu 0}\right) \tilde{A}_{z}-g^{\prime}\left(R_{0}\right) \tilde{B}_{z}+\gamma \tilde{B}_{z z z} \\
-R_{0} \tilde{A}_{z}-\left(U_{\nu 0}-w_{\nu 0}\right) \tilde{B}_{z} \\
-\left(\left(U_{\nu 0}-w_{\nu 0}\right) \tilde{C}\right)_{z}
\end{array}\right)
$$

In order to determine solvability criteria for $(7.21)$ we have to find all $(\tilde{A}, \tilde{B}, \tilde{C})^{T} \in\left(L^{\infty}(\mathbb{R})\right)^{3}$ satisfying

$$
L^{*}(\tilde{A}, \tilde{B}, \tilde{C})^{T}=0 .
$$

Considering the homogeneous problem (7.27) it is well known (cf. [13] for example) that all distributional solutions are already classical solutions. There are five linearly independent solutions of $(7.27)$ in $C^{3}(\mathbb{R}) \times C^{1}(\mathbb{R}) \times C^{1}(\mathbb{R})$. We have to determine whether they are elements of $\left(L^{\infty}(\mathbb{R})\right)^{3}$.

The homogeneous problem (7.27) decouples into one problem for $\tilde{A}$ and $\tilde{B}$ and one for $\tilde{C}$. The latter immediately implies that $\tilde{C}$ has to be some multiple of $R_{0}$, because $J_{0}$ is constant in $z$. The equations for $\tilde{A}$ and $\tilde{B}$ are more involved. They amount to

$$
\begin{aligned}
\frac{j_{0}}{R_{0}} \tilde{A}_{z}+g^{\prime}\left(R_{0}\right) \tilde{B}_{z}-\gamma \tilde{B}_{z z z} & =0, \\
R_{0} \tilde{A}_{z}+\left(U_{\nu 0}-w_{\nu 0}\right) \tilde{B}_{z} & =0 .
\end{aligned}
$$

We can solve (7.29) for $\tilde{A}_{z}$, which yields

$$
\tilde{A}_{z}=-\frac{j_{0}}{R_{0}^{2}} \tilde{B}_{z}
$$

Inserting (7.30) in (7.28) we obtain

$$
-\frac{j_{0}^{2}}{R_{0}^{3}} \tilde{B}_{z}+g^{\prime}\left(R_{0}\right) \tilde{B}_{z}-\gamma \tilde{B}_{z z z}=0 .
$$

For simplification of notation we introduce a new energy density function and a corresponding Gibbs free energy function

$$
\tilde{W}\left(R_{0}\right):=W\left(R_{0}\right)-\frac{1}{2} \frac{j_{0}^{2}}{R_{0}}, \quad \tilde{g}\left(R_{0}\right):=\tilde{W}^{\prime}\left(R_{0}\right)=g\left(R_{0}\right)+\frac{1}{2} \frac{j_{0}^{2}}{R_{0}^{2}} .
$$

Then (7.31) becomes

$$
\tilde{g}^{\prime}\left(R_{0}\right) \tilde{B}_{z}-\gamma \tilde{B}_{z z z}=0 .
$$


We observe that every constant function and $\tilde{B}=R_{0}$, owing to (7.7), are solutions of (7.33). Hence we only have to find one more linearly independent solution of (7.33). When we define $\tilde{D}:=\tilde{B}_{z}$ and $\tilde{E}=\tilde{D}_{z}$, we get from (7.33)

$$
\left(\begin{array}{c}
\tilde{D} \\
\tilde{E}
\end{array}\right)_{z}=\left(\begin{array}{cc}
0 & 1 \\
\frac{1}{\gamma} \tilde{g}^{\prime}\left(R_{0}\right) & 0
\end{array}\right)\left(\begin{array}{c}
\tilde{D} \\
\tilde{E}
\end{array}\right) .
$$

As $\tilde{B}=R_{0}$ is a solution of (7.33), $\left(R_{0, z}, R_{0, z z}\right)^{T}$ is a solution of (7.34). Due to the d'Alembert reduction principle we make the following ansatz:

$$
\begin{aligned}
& \tilde{D}(z)=\varphi(z) R_{0, z}(z), \\
& \tilde{E}(z)=\varphi(z) R_{0, z z}(z)+\psi(z),
\end{aligned}
$$

where the functions $\varphi$ and $\psi$ are to be determined. Inserting (7.35), (7.36) in (7.34) we get

$$
\begin{aligned}
\varphi_{z}(z) R_{0, z}(z)+\varphi(z) R_{0, z z}(z) & =\varphi(z) R_{0, z z}+\psi(z), \\
\varphi_{z}(z) R_{0, z z}(z)+\varphi(z) R_{0, z z z}(z)+\psi_{z}(z) & =\frac{1}{\gamma} \tilde{g}^{\prime}\left(R_{0}\right) \varphi(z) R_{0, z}(z) .
\end{aligned}
$$

We can solve (7.37) with respect to $\varphi_{z}$, which gives

$$
\varphi_{z}=\frac{\psi}{R_{0, z}} .
$$

Using (7.7) and (7.32) in (7.38) we obtain

$$
\psi_{z}(z)=-\varphi_{z}(z) R_{0, z z}(z) \stackrel{(7.39)}{=}-\psi(z) \frac{R_{0, z z}(z)}{R_{0, z}(z)} .
$$

From this we find

for $k \in \mathbb{R}$ and hence by (7.39)

$$
\psi=\frac{k}{R_{0, z}}
$$

$$
\varphi_{z}=\frac{k}{\left(R_{0, z}\right)^{2}} .
$$

We only need one solution $F$ of (7.33) which is linearly independent of the constant and $R_{0}$. Thus we consider the special case

$$
\varphi(z)=\int_{0}^{z} \frac{1}{\left(R_{0, z}(\tilde{z})\right)^{2}} d \tilde{z} .
$$

Then $F$ is given as a primitive function of $\varphi R_{0, z}$. From Lemma 7.3 we conclude that $F \notin L^{\infty}(\mathbb{R})$ as $\left|\varphi(z) R_{0, z}(z)\right| \rightarrow \infty$ for $|z| \rightarrow \infty$. Hence there are only two linearly independent solutions of (7.33) in $L^{\infty}(\mathbb{R})$.

Using (7.30) the general solution of the homogeneous problem $(7.27)$ in $\left(L^{\infty}(\mathbb{R})\right)^{3}$ has the form

$$
\begin{aligned}
& \tilde{A}=k_{1}+k_{2} \frac{j_{0}}{R_{0}}, \\
& \tilde{B}=k_{3}+k_{2} R_{0}, \\
& \tilde{C}=k_{4} R_{0},
\end{aligned}
$$


for arbitrary coefficients $k_{1}, k_{2}, k_{3}, k_{4} \in \mathbb{R}$. This gives rise to the four solvability criteria

$$
\begin{aligned}
\int_{-\infty}^{\infty} f_{1} d z & =0, & \int_{-\infty}^{\infty} f_{2} d z & =0, \\
\int_{-\infty}^{\infty} R_{0} f_{3} d z & =0, & \int_{-\infty}^{\infty} \frac{j_{0}}{R_{0}} f_{1}+R_{0} f_{2} d z & =0 .
\end{aligned}
$$

LEMma 7.5 Let $\left(\rho_{0}, \mathbf{u}_{0}, \rho_{1}, \mathbf{u}_{1}, R_{0}, \mathbf{U}_{0}, R_{1}, \mathbf{U}_{1}\right)$ be an admissible matching solution and let the functions $\Phi, \Psi_{v}, \Psi_{t} \in C^{\infty}(\mathbb{R})$ satisfy

$$
\begin{aligned}
& \Phi(z)=\rho_{1}^{ \pm}+\left(\frac{\partial \rho_{0}}{\partial x^{k}}\right)^{ \pm}\left(r_{1}^{k}+z v_{0}^{k}\right), \\
& \Psi_{\nu}(z)=\left(u_{0}^{j}\right)^{ \pm} v_{1}^{j}+\left(u_{1}^{j}\right)^{ \pm} v_{0}^{j}+\left(\frac{\partial u_{0}^{j}}{\partial x^{k}}\right)^{ \pm} v_{0}^{j}\left(r_{1}^{k}+z v_{0}^{k}\right), \\
& \Psi_{t}(z)=\left(u_{1}^{j}\right)^{ \pm} \frac{t_{0}^{j}}{\left|\mathbf{t}_{0}\right|^{2}}+\left(\frac{\partial u_{0}^{j}}{\partial x^{k}}\right)^{ \pm} \frac{t_{0}^{j}}{\left|\mathbf{t}_{0}\right|^{2}}\left(r_{1}^{k}+z v_{0}^{k}\right)+\left(u_{0}^{j}\right)^{ \pm}\left(\frac{t_{1}^{j}}{\left|\mathbf{t}_{0}\right|^{2}}-2 \frac{t_{0}^{j} t_{0}^{i} t_{1}^{i}}{\left|\mathbf{t}_{0}\right|^{4}}\right),
\end{aligned}
$$

for $z>1$ and $z<-1$ respectively. Further, let $\tilde{R}_{1}=R_{1}-\Phi, \tilde{U}_{v 1}=U_{v 1}-\Psi_{v}$ and $\tilde{U}_{t 1}=U_{t 1}-\Psi_{t}$. Then equations (6.11), (6.12) can be equivalently written as

$$
L\left(\begin{array}{c}
\tilde{R}_{1} \\
\tilde{U}_{\nu 1} \\
\tilde{U}_{t 1}
\end{array}\right)=\left(\begin{array}{c}
f_{1}\left[R_{0}, \mathbf{U}_{0}, \Phi, \Psi\right] \\
f_{2}\left[R_{0}, \mathbf{U}_{0}, \Phi, \Psi\right] \\
f_{3}\left[R_{0}, \mathbf{U}_{0}, \Phi, \Psi\right]
\end{array}\right),
$$

where $L$ is the operator from Lemma 7.4 and

$$
\begin{aligned}
& f_{1}\left[R_{0}, \mathbf{U}_{0}, \Phi, \Psi\right]:=-\frac{1}{\left|\mathbf{t}_{0}\right|}\left(w_{t 0}\left|\mathbf{t}_{0}\right| R_{0}\right)_{s}-R_{0, \tau}+\kappa_{0} R_{0} U_{\nu 0} \\
&-\left(\Phi\left(U_{\nu 0}-w_{\nu 0}\right)+R_{0}\left(\Psi_{\nu}-w_{\nu 1}\right)\right)_{z}, \\
& f_{2}\left[R_{0}, \mathbf{U}_{0}, \Phi, \Psi\right]:=-v_{0}^{j}\left(U_{0}^{j}\right)_{\tau}+(\lambda+2 \mu) \frac{1}{R_{0}}\left(U_{\nu 0}\right)_{z z}-\gamma \kappa_{0} R_{0, z z} \\
&-\left(\left(U_{\nu 0}-w_{\nu 0}\right)\left(\Psi_{\nu}-w_{\nu 1}\right)+g^{\prime}\left(R_{0}\right) \Phi-\gamma \Phi_{z z}\right)_{z}, \\
& f_{3}\left[R_{0}, \mathbf{U}_{0}, \Phi, \Psi\right]:=-\left(\frac{t_{0}^{j}}{\left|\mathbf{t}_{0}\right|^{2}}\right)\left(U_{0}^{j}\right)_{\tau}-\frac{1}{\left|\mathbf{t}_{0}\right|^{2}}\left(g\left(R_{0}\right)-\gamma R_{0, z z}\right)_{s}-\left(U_{\nu 0}-w_{\nu 0}\right)\left(\Psi_{t}\right)_{z} .
\end{aligned}
$$

Proof. We will not consider (6.12) directly but separate it into a normal and a tangential part. The normal part is given by (6.12) times $v_{0}^{j}$ plus (6.9) times $v_{1}^{j}$, i.e.,

$$
\begin{aligned}
-w_{\nu 1}\left(U_{\nu 0}\right)_{z}-w_{\nu 0}\left(U_{\nu 1}\right)_{z}+v_{0}^{j}\left(U_{0}^{j}\right)_{\tau} & +U_{\nu 1}\left(U_{\nu 0}\right)_{z}+U_{\nu 0}\left(U_{\nu 1}\right)_{z}+\left(g^{\prime}\left(R_{0}\right) R_{1}\right)_{z} \\
& =\frac{1}{R_{0}}(\lambda+2 \mu)\left(U_{\nu 0}\right)_{z z}+\gamma R_{1, z z z}-\kappa_{0} \gamma R_{0, z z} .
\end{aligned}
$$


Rearranging the terms in (7.50) gives

$$
\begin{aligned}
\left(\left(U_{\nu 0}-w_{\nu 0}\right)\left(U_{\nu 1}-w_{\nu 1}\right)\right. & \left.+g^{\prime}\left(R_{0}\right) R_{1}\right)_{z} \\
= & -v_{0}^{j}\left(U_{0}^{j}\right)_{\tau}+\frac{1}{R_{0}}(\lambda+2 \mu)\left(U_{\nu 0}\right)_{z z}+\gamma R_{1, z z z}-\kappa_{0} \gamma R_{0, z z}
\end{aligned}
$$

The tangential part of (6.12) is given by (6.12) times $\frac{t_{0}^{j}}{\left|\mathbf{t}_{0}\right|^{2}}$ plus (6.9) times $\frac{t_{1}^{j}}{\left|\mathbf{t}_{0}\right|^{2}}-2 \frac{t_{0}^{j} t_{0}^{i} t_{1}^{i}}{\left|\mathbf{t}_{0}\right|^{4}}$, i.e.,

$$
\begin{aligned}
- & w_{\nu 1}\left(U_{t 0}\right)_{z}-w_{\nu 0}\left(\frac{t_{0}^{j} U_{1}^{j}}{\left|\mathbf{t}_{0}\right|^{2}}\right)_{z}+\frac{t_{0}^{j}}{\left|\mathbf{t}_{0}\right|^{2}}\left(U_{0}^{j}\right)_{\tau}+U_{\nu 1}\left(U_{t 0}\right)_{z} U_{\nu 0}\left(\frac{t_{0}^{j} U_{1}^{j}}{\left|\mathbf{t}_{0}\right|^{2}}\right)_{z} \\
+\frac{t_{0}^{j} v_{1}^{j}}{\left|\mathbf{t}_{0}\right|^{2}} g\left(R_{0}\right)_{z}+ & \frac{1}{\left|\mathbf{t}_{0}\right|^{2}} g\left(R_{0}\right)_{s}+\left(U_{\nu 0}-w_{\nu 0}\right)\left(\frac{t_{1}^{j} U_{0}^{j}}{\left|\mathbf{t}_{0}\right|^{2}}-2 \frac{t_{0}^{j} U_{0}^{j} t_{0}^{i} t_{1}^{i}}{\left|\mathbf{t}_{0}\right|^{4}}\right)_{z}+\frac{t_{1}^{j} v_{0}^{j}}{\left|\mathbf{t}_{0}\right|^{2}} g\left(R_{0}\right)_{z} \\
& =\mu \frac{1}{R_{0}}\left(U_{t 0}\right)_{z z}+\gamma \frac{v_{1}^{j} t_{0}^{j}}{\left|\mathbf{t}_{0}\right|^{2}} R_{0, z z z}+\gamma \frac{1}{\left|\mathbf{t}_{0}\right|^{2}} R_{0, s z z}+\gamma \frac{t_{1}^{j} v_{0}^{i}}{\left|t_{0}\right|^{2}} R_{0, z z z}
\end{aligned}
$$

Using (7.13) and (5.23) this is equivalent to

$$
\left(U_{\nu 0}-w_{\nu 0}\right)\left(U_{t 1}\right)_{z}+\frac{t_{0}^{j}}{\left|\mathbf{t}_{0}\right|^{2}}\left(U_{0}^{j}\right)_{\tau}+\frac{1}{\left|\mathbf{t}_{0}\right|^{2}} g\left(R_{0}\right)_{s}=\gamma \frac{1}{\left|\mathbf{t}_{0}\right|^{2}} R_{0, s z z} .
$$

By the matching conditions, $R_{1}, U_{v 1}, U_{t 1}$ must have the following asymptotic behavior for $z \rightarrow$ $\pm \infty$ :

$$
\begin{aligned}
& R_{1}(z)=\rho_{1}^{ \pm}+\left(\frac{\partial \rho_{0}}{\partial x^{k}}\right)^{ \pm}\left(r_{1}^{k}+z v_{0}^{k}\right)+o(1) \\
& U_{\nu 1}(z)=\left(u_{0}^{j}\right)^{ \pm} v_{1}^{j}+\left(u_{1}^{j}\right)^{ \pm} \nu_{0}^{j}+\left(\frac{\partial u_{0}^{j}}{\partial x^{k}}\right)^{ \pm} v_{0}^{j}\left(r_{1}^{k}+z v_{0}^{k}\right)+o(1) \\
& U_{t 1}(z)=\left(u_{1}^{j}\right)^{ \pm} \frac{t_{0}^{j}}{\left|\mathbf{t}_{0}\right|^{2}}+\left(\frac{\partial u_{0}^{j}}{\partial x^{k}}\right)^{ \pm} \frac{t_{0}^{j}}{\left|\mathbf{t}_{0}\right|^{2}}\left(r_{1}^{k}+z v_{0}^{k}\right)+\left(u_{0}^{j}\right)^{ \pm}\left(\frac{t_{1}^{j}}{\left|\mathbf{t}_{0}\right|^{2}}-2 \frac{t_{0}^{j} t_{0}^{i} t_{1}^{i}}{\left|\mathbf{t}_{0}\right|^{4}}\right)+o(1) .
\end{aligned}
$$

Hence, solving (6.11),(6.12) is equivalent to solving

$$
\begin{aligned}
\left(\tilde{R}_{1}\left(U_{\nu 0}-w_{\nu 0}\right)+R_{0} \tilde{U}_{\nu 1}\right)_{z}= & -\frac{1}{\left|\mathbf{t}_{0}\right|}\left(w_{t 0}\left|\mathbf{t}_{0}\right| R_{0}\right)_{s}-R_{0, \tau}+\kappa_{0} R_{0} U_{\nu 0} \\
& -\left(\Phi\left(U_{\nu 0}-w_{\nu 0}\right)+R_{0}\left(\Psi_{\nu}-w_{\nu 1}\right)\right)_{z}, \\
\left(\left(U_{\nu 0}-w_{\nu 0}\right) \tilde{U}_{\nu 1}+g^{\prime}\left(R_{0}\right) \tilde{R}_{1}-\gamma \tilde{R}_{1, z z}\right)_{z} & =-v_{0}^{j}\left(U_{0}^{j}\right)_{\tau}+(\lambda+2 \mu) \frac{1}{R_{0}}\left(U_{\nu 0}\right)_{z z}-\gamma \kappa R_{0, z z} \\
& -\left(\left(U_{\nu 0}-w_{\nu 0}\right)\left(\Psi_{\nu}-w_{\nu 1}\right)+g^{\prime}\left(R_{0}\right) \Phi-\gamma \Phi_{z z}\right)_{z}
\end{aligned}
$$

and

$$
\left(U_{\nu 0}-w_{\nu 0}\right)\left(\tilde{U}_{t 1}\right)_{z}=-\left(\frac{t_{0}^{j}}{\left|\mathbf{t}_{0}\right|^{2}}\right)\left(U_{0}^{j}\right)_{\tau}-\frac{1}{\left|\mathbf{t}_{0}\right|^{2}}\left(g\left(R_{0}\right)-\gamma R_{0, z z}\right)_{s}-\left(U_{\nu 0}-w_{\nu 0}\right)\left(\Psi_{t}\right)_{z},
$$


with the new boundary conditions

$$
\tilde{R}_{1}(z), \tilde{U}_{\nu 1}(z), \tilde{U}_{t 1}(z) \rightarrow 0 \text { for } z \rightarrow \infty .
$$

In the following we deduce the first order jump conditions in Theorem 6.4 by exploiting the solvability conditions from Lemma 7.4.

\section{$7.3 \quad$ O(1)-order terms}

7.3.1 Mass balance. Relying on Lemma 7.4 and Lemma 7.5 we can determine the first order of the jump of the mass flux across the interface.

LeMma 7.6 Let $\left(\rho_{0}, \mathbf{u}_{0}, \rho_{1}, \mathbf{u}_{1}, R_{0}, \mathbf{U}_{0}, R_{1}, \mathbf{U}_{1}\right)$ be an admissible matching solution then

$$
\llbracket \rho_{\varepsilon}\left(\left(u_{v}\right)_{\varepsilon}-\left(w_{v}\right)_{\varepsilon}\right) \rrbracket_{1}=-\frac{\partial \rho_{\Gamma}}{\partial \tau}-\left(\operatorname{div}_{\Gamma}\left(w_{t 0}\right)-\kappa_{0} w_{\nu 0}\right) \rho_{\Gamma},
$$

where

$$
\rho_{\Gamma}=\int_{0}^{\infty}\left(R_{0}-\rho_{0}^{+}\right) d z-\int_{-\infty}^{0}\left(R_{0}-\rho_{0}^{-}\right) d z+\mathcal{O}(\varepsilon) .
$$

Proof. From Lemma 7.4 and Lemma 7.5 we know

$$
\int_{-\infty}^{\infty} f_{1}\left[R_{0}, \mathbf{U}_{0}, \Phi, \Psi\right] d z=0,
$$

where

$$
\begin{aligned}
f_{1}\left[R_{0}, \mathbf{U}_{0}, \Phi, \Psi\right]=-\frac{1}{\left|\mathbf{t}_{0}\right|}\left(w_{t 0}\left|\mathbf{t}_{0}\right| R_{0}\right)_{s}-R_{0, \tau}+\kappa_{0} R_{0} U_{\nu 0} & \\
& -\left(\Phi\left(U_{\nu 0}-w_{\nu 0}\right)+R_{0}\left(\Psi_{\nu}-w_{\nu 1}\right)\right)_{z},
\end{aligned}
$$

when $f_{1} \in L^{1}(\mathbb{R})$. We start with showing that $f_{1} \in L^{1}(\mathbb{R})$.

We observe that

$$
\left(\rho_{0, t}\right)^{ \pm}+\frac{t_{0}^{i}}{\left|\mathbf{t}_{0}\right|^{2}}\left(\frac{\partial\left(\rho_{0} u_{0}^{i}\right)}{\partial x^{j}}\right)^{ \pm} t_{0}^{j}+v_{0}^{i}\left(\frac{\partial\left(\rho_{0} u_{0}^{i}\right)}{\partial x^{j}}\right)^{ \pm} v_{0}^{j}=0,
$$

because $\rho_{0}, \mathbf{u}_{0}$ satisfy the mass conservation equation in the bulk. Utilizing $w_{\nu 0} v_{0}^{i}+w_{t} t_{0}^{i}=w_{0}^{i}$ equation (7.60) is equivalent to

$$
\begin{aligned}
-w_{\nu 0}\left(\frac{\partial \rho_{0}}{\partial x^{i}}\right)^{ \pm} v_{0}^{i} & -w_{t 0}\left(\frac{\partial \rho_{0}}{\partial x^{i}}\right)^{ \pm} t_{0}^{i}+\left(\rho_{0, t}\right)^{ \pm} \\
& +\left(\frac{\partial \rho_{0}}{\partial x^{i}}\right)^{ \pm} w_{0}^{i}+\frac{t_{0}^{i}}{\left|\mathbf{t}_{0}\right|^{2}}\left(\frac{\partial\left(\rho_{0} u_{0}^{i}\right)}{\partial x^{j}}\right)^{ \pm} t_{0}^{j}+v_{0}^{i}\left(\frac{\partial\left(\rho_{0} u_{0}^{i}\right)}{\partial x^{j}}\right)^{ \pm} v_{0}^{j}=0
\end{aligned}
$$


Using the different forms of the matching conditions implies

$$
-w_{\nu 0}\left(\frac{\partial \rho_{0}}{\partial x^{i}}\right)^{ \pm} v_{0}^{i}-w_{t 0}\left(\rho_{0}^{ \pm}\right)_{s}+\left(\rho_{0}^{ \pm}\right)_{\tau}+\frac{t_{0}^{i}}{\left|\mathbf{t}_{0}\right|}\left(\rho_{0}^{ \pm}\left(u_{0}^{i}\right)^{ \pm}\right)_{s}+v_{0}^{i}\left(\frac{\partial\left(\rho_{0} u_{0}^{i}\right)}{\partial x^{j}}\right)^{ \pm} v_{0}^{j}=0
$$

Due to (5.24) and (7.13) we obtain

$$
\left(u_{\nu 0}^{ \pm}-w_{\nu 0}\right)\left(\frac{\partial \rho_{0}}{\partial x^{i}}\right)^{ \pm} v_{0}^{i}+\rho_{0}^{ \pm}\left(\frac{\partial u_{0}^{i}}{\partial x^{j}}\right)^{ \pm} v_{0}^{i} v_{0}^{j}+\frac{1}{\left|\mathbf{t}_{0}\right|}\left(w_{t 0}\left|\mathbf{t}_{0}\right|\right)_{s} \rho_{0}^{ \pm}+\left(\rho_{0}^{ \pm}\right)_{\tau}-\kappa_{0} \rho_{0}^{ \pm} u_{\nu 0}^{ \pm}=0
$$

Keeping in mind the definition of $f_{1}\left[R_{0}, \mathbf{U}_{0}, \Phi, \Psi\right]$ (see (7.47)), equation (7.63) implies due to (5.31), (5.32) and (5.34)

$$
f_{1}\left[R_{0}(z), \mathbf{U}_{0}(z), \Phi(z), \Psi(z)\right] \rightarrow 0, \quad \text { for }|z| \rightarrow \infty
$$

superlinearly and hence $f_{1}\left[R_{0}, \mathbf{U}_{0}, \Phi, \Psi\right] \in L^{1}(\mathbb{R})$.

Thus we can write (7.22) as

$$
\begin{aligned}
& 0=\lim _{a \rightarrow \infty} {\left[\left(\frac{\partial \rho_{0}}{\partial x^{j}}\right)^{+}\left(v_{0}^{j} a+r_{1}^{j}\right)\left(u_{\nu 0}^{+}-w_{\nu 0}\right)-\left(\frac{\partial \rho_{0}}{\partial x^{j}}\right)^{-}\left(r_{1}^{j}-v_{0}^{j} a\right)\left(u_{\nu 0}^{-}-w_{\nu 0}\right)\right.} \\
&+\rho_{1}^{+}\left(u_{\nu 0}^{+}-w_{\nu 0}\right)-\rho_{1}^{-}\left(u_{\nu 0}^{-}-w_{\nu 0}\right)+\rho_{0}^{+}\left(\frac{\partial u_{0}^{i}}{\partial x^{j}}\right)^{+} v_{0}^{i}\left(v_{0}^{j} a+r_{1}^{j}\right)-\rho_{0}^{-}\left(\frac{\partial u_{0}^{i}}{\partial x^{j}}\right)^{-} v_{0}^{i}\left(r_{1}^{j}-v^{j} a\right) \\
&-w_{\nu 1} \rho_{0}^{+}+w_{\nu 1} \rho_{0}^{-}+\rho_{0}^{+}\left(u_{0}^{j}\right)^{+} v_{1}^{j}+\rho_{0}^{+}\left(u_{1}^{j}\right)^{+} v_{0}^{j}-\rho_{0}^{-}\left(u_{0}^{j}\right)^{-} v_{1}^{j}-\rho_{0}^{-}\left(u_{1}^{j}\right)^{-} v_{0}^{j} \\
&\left.-\int_{-a}^{a}\left(-\frac{1}{\left|\mathbf{t}_{0}\right|}\left(w_{t 0}\left|\mathbf{t}_{0}\right|\right)_{s} R_{0}-R_{0, \tau}+\kappa_{0} R_{0} U_{v 0}\right) d z\right] .
\end{aligned}
$$

Now we would like to decompose the limit into several parts, which have to converge. This can be achieved by subtracting $a$ times $(7.63)^{ \pm}$before letting $a \rightarrow \infty$, i.e.,

$$
\begin{aligned}
& 0=\lim _{a \rightarrow \infty}\left[\left(\frac{\partial \rho_{0}}{\partial x^{j}}\right)^{+} r_{1}^{j}\left(u_{\nu 0}^{+}-w_{\nu 0}\right)-\left(\frac{\partial \rho_{0}}{\partial x^{j}}\right)^{-} r_{1}^{j}\left(u_{\nu 0}^{-}-w_{\nu 0}\right)\right. \\
& \quad+\rho_{1}^{+}\left(u_{\nu 0}^{+}-w_{\nu 0}\right)-\rho_{1}^{-}\left(u_{\nu 0}^{-}-w_{\nu 0}\right)+\rho_{0}^{+}\left(\frac{\partial u_{0}^{i}}{\partial x^{j}}\right)^{+} v_{0}^{i} r_{1}^{j}-\rho_{0}^{-}\left(\frac{\partial u_{0}^{i}}{\partial x^{j}}\right)^{-} v_{0}^{i} r_{1}^{j} \\
& \quad-w_{\nu 1} \rho_{0}^{+}+w_{\nu 1} \rho_{0}^{-}+\rho_{0}^{+}\left(u_{0}^{j}\right)^{+} v_{1}^{j}+\rho_{0}^{+}\left(u_{1}^{j}\right)^{+} v_{0}^{j}-\rho_{0}^{-}\left(u_{0}^{j}\right)^{-} v_{1}^{j}-\rho_{0}^{-}\left(u_{1}^{j}\right)^{-} v_{0}^{j} \\
& \left.-\int_{0}^{a}-\frac{1}{\left|\mathbf{t}_{0}\right|}\left(w_{t 0}\left|\mathbf{t}_{0}\right|\right)_{s}\left(R_{0}-\rho_{0}^{+}\right)-\left(R_{0}-\rho_{0}^{+}\right)_{\tau}+\kappa_{0}\left(R_{0} U_{\nu 0}-\rho_{0}^{+} u_{\nu 0}^{+}\right) d z-\int_{-a}^{0} \ldots d z\right] .
\end{aligned}
$$

Inserting (A.3) in (7.65) and using

$$
R_{0} U_{\nu 0}-\rho_{0}^{+} u_{\nu 0}^{+}=R_{0} w_{\nu 0}-\rho_{0}^{+} w_{\nu 0}
$$


we obtain

$$
\begin{aligned}
& \llbracket\left[\rho_{\varepsilon}\left(\left(u_{\nu}\right)_{\varepsilon}-\left(w_{\nu}\right)_{\varepsilon}\right)\right]_{1} \\
& \quad=\int_{0}^{\infty}\left(-R_{0, \tau}+\left(\rho_{0}^{+}\right)_{\tau}\right) d z+\int_{-\infty}^{0} \ldots \\
& +\int_{0}^{\infty}\left(-\frac{1}{\left|\mathbf{t}_{0}\right|}\left(\left|\mathbf{t}_{0}\right| w_{t 0}\right)_{s} R_{0}+\frac{1}{\left|\mathbf{t}_{0}\right|}\left(\left|\mathbf{t}_{0}\right| w_{t 0}\right)_{s} \rho_{0}^{+}+\kappa_{0} R_{0} w_{\nu 0}-\kappa_{0} \rho_{0}^{+} w_{\nu 0}\right) d z+\int_{-\infty}^{0} \ldots
\end{aligned}
$$

In view of (3.3) we can identify the surface mass density as

$$
\rho_{\Gamma}:=\int_{0}^{\infty}\left(R_{0}-\rho_{0}^{+}\right) d z+\int_{-\infty}^{0}\left(R_{0}-\rho_{0}^{-}\right) d z+\mathcal{O}\left(\varepsilon^{2}\right),
$$

which finishes the proof.

7.3.2 Gibbs free energy. We deduce the first order of the jump of the Gibbs free energy across the interface. The proof is based on Lemma 7.4 and Lemma 7.5.

LEMMA 7.7 Let $\left(\rho_{0}, \mathbf{u}_{0}, \rho_{1}, \mathbf{u}_{1}, R_{0}, \mathbf{U}_{0}, R_{1}, \mathbf{U}_{1}\right)$ be an admissible matching solution. Then

$$
\begin{aligned}
\llbracket \frac{1}{2}\left(\frac{j_{\varepsilon}}{\rho_{\varepsilon}}\right)^{2}+g\left(\rho_{\varepsilon}\right) \prod_{1} & =-\int_{0}^{\infty} v_{0}^{j}\left(U_{0}^{j}-\left(u_{0}^{j}\right)^{+}\right)_{\tau} d z \\
& -\int_{-\infty}^{0} v_{0}^{j}\left(U_{0}^{j}-\left(u_{0}^{j}\right)^{-}\right)_{\tau} d z \\
& -(\lambda+2 \mu) j_{0} \int_{-\infty}^{\infty}\left(\left(\frac{1}{R_{0}}\right)_{z}\right)^{2} d z
\end{aligned}
$$

Proof. From Lemma 7.4 and Lemma 7.5 we know

$$
\int_{-\infty}^{\infty} f_{2}\left[R_{0}, \mathbf{U}_{0}, \Phi, \Psi\right] d z=0
$$

where

$$
\begin{aligned}
f_{2}\left[R_{0}, \mathbf{U}_{0}, \Phi, \Psi\right]= & -v_{0}^{j}\left(U_{0}^{j}\right)_{\tau}+(\lambda+2 \mu) \frac{1}{R_{0}}\left(U_{\nu 0}\right)_{z z}-\gamma \kappa_{0} R_{0, z z} \\
& -\left(\left(U_{\nu 0}-w_{\nu 0}\right)\left(\Psi_{v}-w_{\nu 1}\right)+g^{\prime}\left(R_{0}\right) \Phi-\gamma \Phi_{z z}\right)_{z}
\end{aligned}
$$

when $f_{2} \in L^{1}(\mathbb{R})$. We start with showing that $f_{2} \in L^{1}(\mathbb{R})$.

As the outer quantities $\rho_{0}, \mathbf{u}_{0}$ satisfy (4.5), we have

$$
v_{0}^{j}\left(u_{0, t}^{j}\right)^{ \pm}+\left(u_{0}^{k}\right)^{ \pm}\left(\frac{\partial u_{0}^{j}}{\partial x^{k}}\right)^{ \pm} v_{0}^{j}+g^{\prime}\left(\rho_{0}^{ \pm}\right)\left(\frac{\partial \rho_{0}}{\partial x^{j}}\right)^{ \pm} v_{0}^{j}=0
$$


Due to the matching conditions (5.33), (5.34), (5.37) and the assumptions (7.43), (7.44) and (7.45) on $\Phi, \Psi_{v}$ and $\Psi_{t}$ we get

$$
\begin{array}{r}
f_{2}\left[R_{0}(z), \mathbf{U}_{0}(z), \Phi(z), \Psi(z)\right] \rightarrow-\left(u_{\nu 0}^{ \pm}-w_{\nu 0}\right)\left(\frac{\partial u_{0}^{j}}{\partial x^{k}}\right)^{ \pm} v_{0}^{j} v_{0}^{k}-g^{\prime}\left(\rho_{0}^{ \pm}\right)\left(\frac{\partial \rho_{0}}{\partial x^{k}}\right)^{ \pm} v_{0}^{k} \\
-v_{0}^{j}\left(\frac{\partial u_{0}^{j}}{\partial x^{k}}\right)^{ \pm} w_{0}^{k}-v_{0}^{j}\left(u_{0, t}^{j}\right)^{ \pm}
\end{array}
$$

Inserting (7.70) in (7.71) we get

$$
\begin{array}{r}
f_{2}\left[R_{0}(z), \mathbf{U}_{0}(z), \Phi(z), \Psi(z)\right] \rightarrow\left(\left(u_{0}^{k}\right)^{ \pm}-u_{\nu 0} v_{0}^{k}+w_{\nu 0} v_{0}^{k}-w_{0}^{k}\right)\left(\frac{\partial u_{0}^{j}}{\partial x^{k}}\right)^{ \pm} v_{0}^{k}=0 \\
\text { for }|z| \rightarrow \infty
\end{array}
$$

where the convergence is obtained superlinear, whence $f_{2}\left[R_{0}, \mathbf{U}_{0}, \Phi, \Psi\right] \in L^{1}(\mathbb{R})$.

Due to the matching conditions and (7.43), (7.44) the solvability condition (7.23) is equivalent to

$$
\begin{aligned}
0= & \lim _{a \rightarrow \infty}\left[\left(u_{\nu 0}^{+}-w_{\nu 0}\right) w_{\nu 1}-\left(u_{\nu 0}^{-}-w_{\nu 0}\right) w_{\nu 1}\right. \\
& -\left(u_{\nu 0}^{+}-w_{\nu 0}\right)\left(\left(u_{0}^{j}\right)^{+} v_{1}^{j}+\left(u_{1}^{j}\right)^{+} v_{0}^{j}+\left(\frac{\partial u_{0}^{j}}{\partial x^{k}}\right)^{+} v_{0}^{j}\left(r_{1}^{k}+v_{0}^{k} a\right)\right) \\
& +\left(u_{\nu 0}^{-}-w_{\nu 0}\right)\left(\left(u_{0}^{j}\right)^{-} v_{1}^{j}+\left(u_{1}^{j}\right)^{-} v_{0}^{j}+\left(\frac{\partial u_{0}^{j}}{\partial x^{k}}\right)^{-} v_{0}^{j}\left(r_{1}^{k}-v_{0}^{k} a\right)\right) \\
& -g^{\prime}\left(\rho_{0}^{+}\right) \rho_{1}^{+}-g^{\prime}\left(\rho_{0}^{+}\right)\left(\frac{\partial \rho_{0}}{\partial x^{k}}\right)^{+}\left(r_{1}^{k}+v_{0}^{k} a\right)+g^{\prime}\left(\rho_{0}^{-}\right) \rho_{1}^{-} \\
& \left.+g^{\prime}\left(\rho_{0}^{-}\right)\left(\frac{\partial \rho_{0}}{\partial x^{k}}\right)^{-}\left(r_{1}^{k}-v_{0}^{k} a\right)-\int_{-a}^{a} v_{0}^{j}\left(U_{0}^{j}\right)_{\tau} d z+(\lambda+2 \mu) \int_{-a}^{a} \frac{1}{R_{0}}\left(U_{\nu 0}\right)_{z z} d z\right] .
\end{aligned}
$$

Now we subtract $a$ times $(7.70)^{+}$and $a$ times (7.70) - before letting $a \rightarrow \infty$ and obtain

$$
\begin{aligned}
0= & \lim _{a \rightarrow \infty}\left[\left(u_{\nu 0}^{+}-w_{\nu 0}\right) w_{\nu 1}-\left(u_{\nu 0}^{-}-w_{\nu 0}\right) w_{\nu 1}\right. \\
& -\left(u_{\nu 0}^{+}-w_{\nu 0}\right) \llbracket \psi_{0} \rrbracket\left(\left(u_{0}^{j}\right)^{+} v_{1}^{j}+\left(u_{1}^{j}\right)^{+} v_{0}^{j}+\left(\frac{\partial u_{0}^{j}}{\partial x^{k}}\right)^{+} v_{0}^{j} r_{1}^{k}\right) \\
& +\left(u_{\nu 0}^{-}-w_{\nu 0}\right)\left(\left(u_{0}^{j}\right)^{-} v_{1}^{j}+\left(u_{1}^{j}\right)^{-} v_{0}^{j}+\left(\frac{\partial u_{0}^{j}}{\partial x^{k}}\right)^{-} v_{0}^{j} r_{1}^{k}\right) \\
& -g^{\prime}\left(\rho_{0}^{+}\right) \rho_{1}^{+}-g^{\prime}\left(\rho_{0}^{+}\right)\left(\frac{\partial \rho_{0}}{\partial x^{k}}\right)^{+} r_{1}^{k}+g^{\prime}\left(\rho_{0}^{-}\right) \rho_{1}^{-}+g^{\prime}\left(\rho_{0}^{-}\right)\left(\frac{\partial \rho_{0}}{\partial x^{k}}\right)^{-} r_{1}^{k} \\
& \left.-\int_{0}^{a} v_{0}^{j}\left(U_{0}^{j}-\left(u_{0}^{j}\right)^{+}\right)_{\tau} d z-\int_{-a}^{0} \ldots d z+(\lambda+2 \mu) \int_{-a}^{a} \frac{1}{R_{0}}\left(U_{\nu 0}\right)_{z z} d z\right] .
\end{aligned}
$$


Using (A.5) equation (7.74) means

$$
\begin{aligned}
\llbracket \frac{1}{2}\left(\left(u_{v}\right)_{\varepsilon}-\left(w_{v}\right)_{\varepsilon}\right)^{2}+g\left(\rho_{\varepsilon}\right) \rrbracket_{1}= & -\int_{0}^{\infty} v_{0}^{j}\left(\left(U_{0}^{j}\right)_{\tau}(z)-\left(\left(u_{0}^{j}\right)^{+}\right)_{\tau}\right) d z \\
& -\int_{-\infty}^{0} v_{0}^{j}\left(\left(U_{0}^{j}\right)_{\tau}(z)-\left(\left(u_{0}^{j}\right)^{-}\right)_{\tau}\right) d z \\
& +\int_{-\infty}^{\infty}(\lambda+2 \mu) \frac{1}{R_{0}(z)}\left(U_{\nu 0}\right)_{z z}(z) d z
\end{aligned}
$$

Inserting $U_{\nu 0}=\frac{J_{0}}{R_{0}}+w_{\nu}$ in (7.75) yields

$$
\begin{aligned}
\llbracket \frac{1}{2}\left(\left(u_{v}\right)_{\varepsilon}-\left(w_{v}\right)_{\varepsilon}\right)^{2}+g\left(\rho_{\varepsilon}\right) \rrbracket_{1}= & -\int_{0}^{\infty} v_{0}^{j}\left(U_{0}^{j}(z)-\left(u_{0}^{j}\right)^{+}\right)_{\tau} d z \\
& -\int_{-\infty}^{0} v_{0}^{j}\left(U_{0}^{j}(z)-\left(u_{0}^{j}\right)^{-}\right)_{\tau} d z \\
& -\int_{-\infty}^{\infty}(\lambda+2 \mu) J_{0}\left(\left(\frac{1}{R_{0}}\right)_{z}\right)^{2} d z .
\end{aligned}
$$

7.3.3 Momentum balance. Using the last two solvability conditions from Lemma 7.4 together with Lemma 7.5, we will determine the first order of the pressure jump across the interface.

LEMmA 7.8 Let $\left(\rho_{0}, \mathbf{u}_{0}, \rho_{1}, \mathbf{u}_{1}, R_{0}, \mathbf{U}_{0}, R_{1}, \mathbf{U}_{1}\right)$ be an admissible matching solution. Then

$$
\llbracket \rho_{\varepsilon}\left(\left(u_{\nu}\right)_{\varepsilon}-\left(w_{\nu}\right)_{\varepsilon}\right)\left(\mathbf{u}_{\varepsilon}-\mathbf{w}_{\varepsilon}\right)+p\left(\rho_{\varepsilon}\right) \boldsymbol{v}_{\varepsilon} \rrbracket_{1}=-\rho_{\Gamma} \frac{\partial \mathbf{w}_{0}}{\partial \tau}+\operatorname{div}_{\Gamma}\left(\sigma_{\Gamma}\right)
$$

where

$$
\begin{aligned}
& \rho_{\Gamma}=\int_{0}^{\infty}\left(R_{0}-\rho_{0}^{+}\right) d z-\int_{-\infty}^{0}\left(R_{0}-\rho_{0}^{-}\right) d z+\mathcal{O}(\varepsilon), \\
& \sigma_{\Gamma}^{j}=\left(\int_{0}^{\infty}\left(p\left(\rho_{0}^{+}\right)-p\left(R_{0}\right)-\frac{\gamma}{2}\left(R_{0, z}\right)^{2}\right) d z+\int_{-\infty}^{0} \ldots\right) \frac{t_{0}^{j}}{\left|\mathbf{t}_{0}\right|^{2}}+\mathcal{O}(\varepsilon) .
\end{aligned}
$$

Proof. From Lemma 7.4 and Lemma 7.5 we have the solvability criteria

$$
\int_{-\infty}^{\infty} R_{0} f_{3} d z=0, \quad \int_{-\infty}^{\infty} \frac{j_{0}}{R_{0}} f_{1}+R_{0} f_{2} d z=0
$$


where

$$
\begin{aligned}
& f_{1}\left[R_{0}, \mathbf{U}_{0}, \Phi, \Psi\right]=-\frac{1}{\left|\mathbf{t}_{0}\right|}\left(w_{t 0}\left|\mathbf{t}_{0}\right| R_{0}\right)_{s}-R_{0, \tau}+\kappa_{0} R_{0} U_{\nu 0} \\
& -\left(\Phi\left(U_{\nu 0}-w_{\nu 0}\right)+R_{0}\left(\Psi_{\nu}-w_{\nu 1}\right)\right)_{z}, \\
& f_{2}\left[R_{0}, \mathbf{U}_{0}, \Phi, \Psi\right]=-v_{0}^{j}\left(U_{0}^{j}\right)_{\tau}+(\lambda+2 \mu) \frac{1}{R_{0}}\left(U_{\nu 0}\right)_{z z}-\gamma \kappa_{0} R_{0, z z} \\
& -\left(\left(U_{\nu 0}-w_{\nu 0}\right)\left(\Psi_{\nu}-w_{\nu 1}\right)+g^{\prime}\left(R_{0}\right) \Phi-\gamma \Phi_{z z}\right)_{z}, \\
& f_{3}\left[R_{0}, \mathbf{U}_{0}, \Phi, \Psi\right]=-\left(\frac{t_{0}^{j}}{\left|\mathbf{t}_{0}\right|^{2}}\right)\left(U_{0}^{j}\right)_{\tau}-\frac{1}{\left|\mathbf{t}_{0}\right|^{2}}\left(g\left(R_{0}\right)-\gamma R_{0, z z}\right)_{s}-\left(U_{\nu 0}-w_{\nu 0}\right)\left(\Psi_{t}\right)_{z},
\end{aligned}
$$

when $f_{1}, f_{2}, f_{3} \in L^{1}(\mathbb{R})$. From the proofs of Lemma 7.6 and Lemma 7.7 we already know that $f_{1}, f_{2} \in L^{1}(\mathbb{R})$. It remains to show that $f_{3} \in L^{1}(\mathbb{R})$.

By the matching conditions and (7.45) we get

$$
\begin{aligned}
f_{3} \rightarrow-\frac{t_{0}^{j}}{\left|\mathbf{t}_{0}\right|^{2}}\left(\frac{\partial u_{0}^{j}}{\partial x^{k}}\right)^{ \pm} w_{0}^{k} & -\frac{t_{0}^{j}}{\left|\mathbf{t}_{0}\right|^{2}}\left(\frac{\partial u_{0}^{j}}{\partial t}\right)^{ \pm} \\
& -\frac{t_{0}^{k}}{\left|\mathbf{t}_{0}\right|^{2}}\left(\frac{\partial g\left(\rho_{0}\right)}{\partial x^{k}}\right)^{ \pm}-\left(u_{\nu 0}^{ \pm}-w_{\nu 0}\right)-\frac{t_{0}^{j}}{\left|\mathbf{t}_{0}\right|^{2}}\left(\frac{\partial u_{0}^{j}}{\partial x^{k}}\right)^{ \pm} v_{0}^{k}
\end{aligned}
$$

On the other hand, $\rho_{0}, \mathbf{u}_{0}$ satisfy (4.5) in the bulk, such that

$$
\frac{t_{0}^{j}}{\left|\mathbf{t}_{0}\right|^{2}}\left(\left(\frac{\partial u_{0}^{j}}{\partial t}\right)^{ \pm}+\left(u_{0}^{k}\right)^{ \pm}\left(\frac{\partial u_{0}^{j}}{\partial x^{k}}\right)^{ \pm}+\left(\frac{\partial g\left(\rho_{0}\right)}{\partial x^{j}}\right)^{ \pm}\right)=0 .
$$

Inserting (7.81) in (7.80) we get

$$
f_{3} \rightarrow-\frac{t_{0}^{j}}{\left|\mathbf{t}_{0}\right|^{2}}\left(\left(u_{0}^{k}\right)^{ \pm}-w_{0}^{k}-\left(u_{\nu 0}^{ \pm}-w_{\nu 0}\right) v_{0}^{k}\right)\left(\frac{\partial u_{0}^{j}}{\partial x^{k}}\right)^{ \pm}=0 .
$$

To obtain a solvability criterion, which has the form of the Young-Laplace law, we will deal with the solvability criteria (7.24) and (7.25) simultaneously. As $\boldsymbol{v}_{0}$ and $\mathbf{t}_{0}$ are linearly independent equations (7.24) and (7.25) are equivalent to

$$
\int_{-\infty}^{\infty} R_{0} f_{3}\left[R_{0}, \mathbf{U}_{0}, \Phi, \Psi\right] t_{0}^{i}+\frac{j_{0}}{R_{0}} f_{1}\left[R_{0}, \mathbf{U}_{0}, \Phi, \Psi\right] v_{0}^{i}+R_{0} f_{2}\left[R_{0}, \mathbf{U}_{0}, \Phi, \Psi\right] v_{0}^{i} d z=0
$$

As $f_{1}, f_{2}, f_{3}$ go to zero superlinearly for $|z| \rightarrow \infty$, we have

$$
\lim _{z \rightarrow \pm \infty} R_{0} f_{3}\left[R_{0}, \mathbf{U}_{0}, \Phi, \Psi\right] t^{i}+\frac{j_{0}}{R_{0}} f_{1}\left[R_{0}, \mathbf{U}_{0}, \Phi, \Psi\right] v^{i}+R_{0} f_{2}\left[R_{0}, \mathbf{U}_{0}, \Phi, \Psi\right] v^{i}=0 .
$$


Inserting (7.47)-(7.49) in (7.83) yields

$$
\begin{aligned}
0=\int_{-\infty}^{\infty}\left[\frac{J_{0}}{R_{0}} v_{0}^{i}(\right. & \left.-\frac{1}{\left|\mathbf{t}_{0}\right|}\left(w_{t 0}\left|\mathbf{t}_{0}\right|\right)_{s} R_{0}-R_{0, \tau}+\kappa_{0} R_{0} U_{\nu 0}-\left(\Phi \frac{J_{0}}{R_{0}}+R_{0}\left(\Psi_{\nu}-w_{\nu 1}\right)\right)_{z}\right) \\
+ & R_{0} v_{0}^{i}\left(-v_{0}^{j}\left(U_{0}^{j}\right)_{\tau}+(\lambda+2 \mu) \frac{1}{R_{0}}\left(U_{\nu 0}\right)_{z z}-\gamma \kappa_{0} R_{0, z z}\right) \\
& -R_{0} v_{0}^{i}\left(\frac{J_{0}}{R_{0}}\left(\Psi_{\nu}-w_{\nu 1}\right)+g^{\prime}\left(R_{0}\right) \Phi-\gamma \Phi_{z z}\right)_{z} \\
& \left.-R_{0} t_{0}^{i}\left(\frac{t_{0}^{j}}{\left|\mathbf{t}_{0}\right|^{2}}\left(U_{0}^{j}\right)_{\tau}+\frac{1}{\left|\mathbf{t}_{0}\right|^{2}}\left(g\left(R_{0}\right)-\gamma R_{0, z z}\right)_{s}+\frac{J_{0}}{R_{0}}\left(\Psi_{t}\right)_{z}\right)\right] d z
\end{aligned}
$$

The terms containing $w_{\nu 1}$ cancel out and using integration by parts, (7.85) becomes

$$
\begin{aligned}
0=\int_{-\infty}^{\infty} & {\left[-J_{0} v_{0}^{i} \frac{1}{\left|\mathbf{t}_{0}\right|}\left(w_{t 0}\left|\mathbf{t}_{0}\right|\right)_{s}-\frac{J_{0}}{R_{0}} v_{0}^{i} R_{0, \tau}+\kappa_{0} v_{0}^{i} J_{0} U_{\nu 0}\right.} \\
- & v_{0}^{i}\left(J_{0} \Psi_{\nu}+\frac{J_{0}^{2}}{R_{0}^{2}} \Phi+J_{0} \Psi_{\nu}+p^{\prime}\left(R_{0}\right) \Phi-\gamma\left(\Phi_{z z} R_{0}-\Phi_{z} R_{0, z}+\Phi R_{0, z z}\right)\right)_{z} \\
+ & \frac{J_{0}}{R_{0}}\left(\frac{J_{0}}{R_{0}}\right)_{z} v_{0}^{i} \Phi+\left(\frac{J_{0}}{R_{0}}\right)_{z} v_{0}^{i} R_{0} \Psi_{\nu}-R_{0}\left(U_{0}^{i}\right)_{\tau}+(\lambda+2 \mu)\left(U_{\nu 0}\right)_{z z} v_{0}^{i} \\
& -\gamma \kappa_{0} v_{0}^{i} R_{0} R_{0, z z}+v_{0}^{i} R_{0, z} \frac{J_{0}}{R_{0}} \Psi_{\nu}+g\left(R_{0}\right)_{z} \Phi v_{0}^{i}-\gamma R_{0, z z z} \Phi v_{0}^{i} \\
& \left.-\frac{t_{0}^{i}}{\left|\mathbf{t}_{0}\right|^{2}} p\left(R_{0}\right)_{s}+\gamma \frac{t_{0}^{i}}{\left|\mathbf{t}_{0}\right|^{2}} R_{0} R_{0, s z z}-J_{0} t_{0}^{i}\left(\Psi_{t}\right)_{z}\right] d z
\end{aligned}
$$

We observe that (6.9) and (7.13) imply

$$
\frac{J_{0}}{R_{0}} v_{0}^{i} R_{0, \tau}+R_{0}\left(U_{0}^{i}\right)_{\tau}=\left(J_{0} v_{0}^{i}\right)_{\tau}+R_{0}\left(w_{0}^{i}\right)_{\tau} \quad \text { and } \quad\left(\frac{J_{0}}{R_{0}}\right)_{z} R_{0}+\left(\frac{J_{0}}{R_{0}}\right) R_{0, z}=0
$$

Using this (7.86) yields

$$
\begin{aligned}
0=\int_{-\infty}^{\infty}\left[-J_{0} v_{0}^{i} \frac{1}{\left|\mathbf{t}_{0}\right|}\left(w_{t 0}\left|\mathbf{t}_{0}\right|\right)_{s}-\left(J_{0} v_{0}^{i}\right)_{\tau}-R_{0}\left(w_{0}^{i}\right)_{\tau}+\kappa_{0} v_{0}^{i} J_{0} U_{v 0}\right. \\
\quad-v_{0}^{i}\left(2 J_{0} \Psi_{v}+\frac{J_{0}^{2}}{R_{0}^{2}} \Phi+p^{\prime}\left(R_{0}\right) \Phi-\gamma\left(\Phi_{z z} R_{0}-\Phi_{z} R_{0, z}+\Phi R_{0, z z}\right)\right)_{z} \\
+(\lambda+2 \mu)\left(U_{v 0}\right)_{z z} v_{0}^{i}-\gamma \kappa_{0} v_{0}^{i} R_{0} R_{0, z z}-\frac{t_{0}^{i}}{\left|\mathbf{t}_{0}\right|^{2}} p\left(R_{0}\right)_{s}+\gamma \frac{t_{0}^{i}}{\left|\mathbf{t}_{0}\right|^{2}} R_{0} R_{0, s z z}-J_{0} t_{0}^{i}\left(\Psi_{t}\right)_{z} \\
\left.+\Phi v_{0}^{i}\left(\frac{J_{0}}{R_{0}}\left(\frac{J_{0}}{R_{0}}\right)_{z}+g\left(R_{0}\right)_{z}-\gamma R_{0, z z z}\right)\right] d z
\end{aligned}
$$

We remark that the last line of (7.87) vanishes due to (6.10). 
Now we will consider some of the terms from (7.87) separately. We start with multiplying (6.10) with $R_{0}$ and subtracting (6.9) times $w_{0}^{j}$. We obtain

$$
\left(R_{0} U_{\nu 0}\left(U_{0}^{j}-w_{0}^{j}\right)\right)_{z}-\underbrace{\left(R_{0} w_{\nu 0}\left(U_{0}^{j}-w_{0}^{j}\right)\right)_{z}}_{=0}=-v_{0}^{j} p\left(R_{0}\right)_{z}+v_{0}^{j} \gamma R_{0} R_{0, z z z} .
$$

So there exists $D_{1}^{i}$ independent of $z$ such that

$$
v_{0}^{i} j_{0} U_{\nu 0}=-v_{0}^{i} p\left(R_{0}\right)+\gamma v_{0}^{i} R_{0} R_{0, z z}-\gamma v_{0}^{i} \frac{1}{2}\left(R_{0, z}\right)^{2}+D_{1}^{i}
$$

With (5.3) we compute

$$
\begin{aligned}
p\left(R_{0}\right)_{s} \frac{t_{0}^{j}}{\left|\mathbf{t}_{0}\right|^{2}} & =\frac{1}{\left|\mathbf{t}_{0}\right|}\left(p\left(R_{0}\right) \frac{t_{0}^{j}}{\left|\mathbf{t}_{0}\right|}\right)_{s}-p\left(R_{0}\right) \frac{1}{\left|\mathbf{t}_{0}\right|}\left(\frac{t_{0}^{j}}{\left|\mathbf{t}_{0}\right|}\right)_{s} \\
& =\frac{1}{\left|\mathbf{t}_{0}\right|}\left(\frac{t_{0}^{j}}{\left|\mathbf{t}_{0}\right|} p\left(R_{0}\right)\right)_{s}-v_{0}^{j} \kappa_{0} p\left(R_{0}\right) .
\end{aligned}
$$

Additionally we observe that due to (6.9)

$$
D_{2}^{j}:=\left(R_{0}\left(U_{0}^{j}-w_{0}^{j}\right)\right)_{\tau}=\left(j_{0} v_{0}^{j}\right)_{\tau}
$$

and

$$
D_{3}^{j}:=\frac{1}{\left|\mathbf{t}_{0}\right|}\left(\left|\mathbf{t}_{0}\right| w_{t 0}\right)_{s} R_{0}\left(U_{0}^{j}-w_{0}^{j}\right)=\frac{1}{\left|\mathbf{t}_{0}\right|}\left(\left|\mathbf{t}_{0}\right| w_{t 0}\right)_{s} j_{0} v_{0}^{j}
$$

are independent of $z$. Now we insert (7.89) and (7.90) in (7.87) and we end up with

$$
\begin{aligned}
0= & \int_{-\infty}^{\infty}\left[-D_{3}^{i}-D_{2}^{i}-R_{0}\left(w_{0}^{i}\right)_{\tau}-\kappa_{0} \gamma v_{0}^{i} \frac{1}{2}\left(R_{0, z}\right)^{2}+\kappa_{0} D_{1}^{i}\right. \\
-\left(2 J_{0} \Psi_{\nu} v_{0}^{i}\right. & \left.+J_{0} \Psi_{t} t_{0}^{i}+v_{0}^{i} \frac{J_{0}^{2}}{R_{0}^{2}} \Phi+p^{\prime}\left(R_{0}\right) \Phi v_{0}^{i}-\gamma v_{0}^{i}\left(\Phi_{z z} R_{0}-\Phi_{z} R_{0, z}+\Phi R_{0, z z}\right)\right)_{z} \\
& \left.+(\lambda+2 \mu)\left(U_{\nu 0}\right)_{z z} v_{0}^{i}-\frac{1}{\left|\mathbf{t}_{0}\right|}\left(\frac{t_{0}^{i}}{\left|\mathbf{t}_{0}\right|} p\left(R_{0}\right)\right)_{s}+\gamma \frac{t_{0}^{i}}{\left|\mathbf{t}_{0}\right|^{2}} R_{0} R_{0, s z z}\right] d z
\end{aligned}
$$

By (7.84) the integrand in (7.93) goes to zero for $|z| \rightarrow \infty$, i.e.,

$$
\begin{aligned}
0=-D_{2}^{i}- & D_{3}^{i}+\kappa_{0} D_{1}^{i}-\rho_{0}^{ \pm}\left(w_{0}^{i}\right)_{\tau}-2 j_{0}\left(\frac{\partial u_{0}^{j}}{\partial x^{k}}\right)^{ \pm} v_{0}^{i} v_{0}^{j} v_{0}^{k}-j_{0}\left(\frac{\partial u_{0}^{j}}{\partial x^{k}}\right)^{ \pm} \frac{t_{0}^{i} t_{0}^{j}}{\left|\mathbf{t}_{0}\right|^{2}} v_{0}^{k} \\
& -\frac{j_{0}^{2}}{\left(\rho_{0}^{ \pm}\right)^{2}} v_{0}^{i}\left(\frac{\partial \rho_{0}}{\partial x^{k}}\right)^{ \pm} v_{0}^{k}-p^{\prime}\left(\rho_{0}^{ \pm}\right) v_{0}^{i}\left(\frac{\partial \rho_{0}}{\partial x^{k}}\right)^{ \pm} v_{0}^{k}-\frac{1}{\left|\mathbf{t}_{0}\right|}\left(p\left(\rho_{0}^{ \pm}\right) \frac{t_{0}^{i}}{\left|\mathbf{t}_{0}\right|}\right)_{s}
\end{aligned}
$$


Using the matching conditions and (7.43)-(7.45) equation (7.93) is equivalent to

$$
\begin{aligned}
0= & \lim _{a \rightarrow \infty}\left[-2 a D_{2}^{i}-2 a D_{3}^{i}+2 a \kappa_{0} D_{1}^{i}-\int_{-a}^{a} R_{0}\left(w_{0}^{i}\right)_{\tau}+\frac{\gamma}{2} \kappa_{0} v_{0}^{i}\left(R_{0, z}\right)^{2} d z\right. \\
& -2 j_{0} v_{0}^{i}\left(\left(u_{0}^{j}\right)^{+} v_{1}^{j}+\left(u_{1}^{j}\right)^{+} v_{0}^{j}+\left(\frac{\partial u_{0}^{j}}{\partial x^{k}}\right)^{+} v_{0}^{j}\left(r_{1}^{k}+a v_{0}^{k}\right)\right) \\
& +2 j_{0} v_{0}^{i}\left(\left(u_{0}^{j}\right)^{-} v_{1}^{j}+\left(u_{1}^{j}\right)^{-} v_{0}^{j}+\left(\frac{\partial u_{0}^{j}}{\partial x^{k}}\right)^{-} v_{0}^{j}\left(r_{1}^{k}-a v_{0}^{k}\right)\right) \\
& -j_{0} t_{0}^{i}\left(\left(u_{1}^{j}\right)^{+} \frac{t_{0}^{j}}{\left|\mathbf{t}_{0}\right|^{2}}+\left(\frac{\partial u_{0}^{j}}{\partial x^{k}}\right)^{+} \frac{t_{0}^{j}}{\left|\mathbf{t}_{0}\right|^{2}}\left(r_{1}^{k}+a v_{0}^{k}\right)+\left(u_{0}^{k}\right)^{+}\left(\frac{t_{1}^{k}}{\left|\mathbf{t}_{0}\right|^{2}}-2 \frac{t_{0}^{k} t_{0}^{j} t_{1}^{j}}{\left|\mathbf{t}_{0}\right|^{4}}\right)\right) \\
& +j_{0} t_{0}^{i}\left(\left(u_{1}^{j}\right)^{-} \frac{t_{0}^{j}}{\left|\mathbf{t}_{0}\right|^{2}}+\left(\frac{\partial u_{0}^{j}}{\partial x^{k}}\right)^{-} \frac{t_{0}^{j}}{\left|\mathbf{t}_{0}\right|^{2}}\left(r_{1}^{k}-a v_{0}^{k}\right)+\left(u_{0}^{k}\right)^{-}\left(\frac{t_{1}^{k}}{\left|\mathbf{t}_{0}\right|^{2}}-2 \frac{t_{0}^{k} t_{0}^{j} t_{1}^{j}}{\left|\mathbf{t}_{0}\right|^{4}}\right)\right) \\
& -v_{0}^{i}\left(\frac{j_{0}^{2}}{\left(\rho_{0}^{+}\right)^{2}}+p^{\prime}\left(\rho_{0}^{+}\right)\right)\left(\rho_{1}^{+}+\left(\frac{\partial \rho_{0}}{\partial x^{k}}\right)^{+}\left(r_{1}^{k}+a v_{0}^{k}\right)\right) \\
& +v_{0}^{i}\left(\frac{j_{0}^{2}}{\left(\rho_{0}^{-}\right)^{2}}+p^{\prime}\left(\rho_{0}^{-}\right)\right)\left(\rho_{1}^{-}+\left(\frac{\partial \rho_{0}}{\partial x^{k}}\right)^{-}\left(r_{1}^{k}-a v_{0}^{k}\right)\right) \\
& \left.-\int_{-a}^{a} \frac{1}{\left|\mathbf{t}_{0}\right|}\left(p\left(R_{0}\right) \frac{t_{0}^{i}}{\left|\mathbf{t}_{0}\right|}\right){ }_{s}-\gamma \frac{t_{0}^{i}}{\left|\mathbf{t}_{0}\right|^{2}} R_{0} R_{0, s z z} d z\right] .
\end{aligned}
$$

We subtract $a$ times (7.94) ${ }^{+}$and $a$ times (7.94) $)^{-}$before letting $a \rightarrow \infty$ which yields

$$
\begin{aligned}
0= & -\int_{0}^{\infty}\left(R_{0}-\rho_{0}^{+}\right)\left(w_{0}^{i}\right)_{\tau}+\frac{\gamma}{2} \kappa_{0} v_{0}^{i}\left(R_{0, z}\right)^{2} d z-\int_{-\infty}^{0} \ldots d z \\
& -2 j_{0} v_{0}^{i}\left(\left(u_{0}^{j}\right)^{+} v_{1}^{j}+\left(u_{1}^{j}\right)^{+} v_{0}^{j}+\left(\frac{\partial u_{0}^{j}}{\partial x^{k}}\right)^{+} v_{0}^{j} r_{1}^{k}\right) \\
& +2 j_{0} v_{0}^{i}\left(\left(u_{0}^{j}\right)^{-} v_{1}^{j}+\left(u_{1}^{j}\right)^{-} v_{0}^{j}+\left(\frac{\partial u_{0}^{j}}{\partial x^{k}}\right)^{-} v_{0}^{j} r_{1}^{k}\right) \\
& -j_{0} t_{0}^{i}\left(\left(u_{1}^{j}\right)^{+} \frac{t_{0}^{j}}{\left|\mathbf{t}_{0}\right|^{2}}+\left(\frac{\partial u_{0}^{j}}{\partial x^{k}}\right)^{+} \frac{t_{0}^{j}}{\left|\mathbf{t}_{0}\right|^{2}} r_{1}^{k}+\left(u_{0}^{k}\right)^{+}\left(\frac{t_{1}^{k}}{\left|\mathbf{t}_{0}\right|^{2}}-2 \frac{t_{0}^{k} t_{0}^{j} t_{1}^{j}}{\left|\mathbf{t}_{0}\right|^{4}}\right)\right) \\
& +j_{0} t_{0}^{i}\left(\left(u_{1}^{j}\right)^{-} \frac{t_{0}^{j}}{\left|\mathbf{t}_{0}\right|^{2}}+\left(\frac{\partial u_{0}^{j}}{\partial x^{k}}\right)^{-} \frac{t_{0}^{j}}{\left|\mathbf{t}_{0}\right|^{2}} r_{1}^{k}+\left(u_{0}^{k}\right)^{-}\left(\frac{t_{1}^{k}}{\left|\mathbf{t}_{0}\right|^{2}}-2 \frac{t_{0}^{k} t_{0}^{j} t_{1}^{j}}{\left|\mathbf{t}_{0}\right|^{4}}\right)\right) \\
& -v_{0}^{i}\left(\frac{j_{0}^{2}}{\left(\rho_{0}^{+}\right)^{2}}+p^{\prime}\left(\rho_{0}^{+}\right)\right)\left(\rho_{1}^{+}+\left(\frac{\partial \rho_{0}}{\partial x^{k}}\right)^{+} r_{1}^{k}\right) \\
& +v_{0}^{i}\left(\frac{j_{0}^{2}}{\left(\rho_{0}^{-}\right)^{2}}+p^{\prime}\left(\rho_{0}^{-}\right)\right)\left(\rho_{1}^{-}+\left(\frac{\partial \rho_{0}}{\partial x^{k}}\right)^{-} r_{1}^{k}\right)
\end{aligned}
$$




$$
-\int_{0}^{\infty} \frac{1}{\left|\mathbf{t}_{0}\right|}\left(\left(p\left(R_{0}\right)-p\left(\rho_{0}^{+}\right)\right) \frac{t_{0}^{i}}{\left|\mathbf{t}_{0}\right|}\right)_{s}-\gamma \frac{t_{0}^{i}}{\left|\mathbf{t}_{0}\right|^{2}} R_{0} R_{0, s z z} d z-\int_{-\infty}^{0} \ldots d z .
$$

As explained in the appendix, (7.96) can be written as

$$
\begin{aligned}
& \llbracket \rho_{\varepsilon}\left(\left(u_{v}\right)_{\varepsilon}-\left(w_{v}\right)_{\varepsilon}\right)\left(\mathbf{u}_{\varepsilon}-\mathbf{w}_{\varepsilon}\right)+p\left(\rho_{\varepsilon}\right) \boldsymbol{v}_{\varepsilon} \rrbracket_{1}= \\
& \quad-\left(w_{0}^{j}\right)_{\tau} \int_{0}^{\infty}\left(R_{0}-\rho_{0}^{+}\right) d z-\int_{-\infty}^{0} \ldots d z \\
& -\int_{0}^{\infty}\left[\frac{1}{\left|\mathbf{t}_{0}\right|}\left(p\left(R_{0}\right) \frac{t_{0}^{j}}{\left|\mathbf{t}_{0}\right|}\right)_{s}-\frac{1}{\left|\mathbf{t}_{0}\right|}\left(p\left(\rho_{0}^{+}\right) \frac{t_{0}^{j}}{\left|\mathbf{t}_{0}\right|}\right)_{s}\right] d z-\int_{-\infty}^{0} \ldots d z \\
& \quad-\kappa_{0} \gamma \frac{1}{2} v_{0}^{j} \int_{-\infty}^{\infty}\left(R_{0, z}\right)^{2} d z-\gamma \frac{1}{2} \frac{t_{0}^{j}}{\left|\mathbf{t}_{0}\right|^{2}} \int_{-\infty}^{\infty}\left(\left(R_{0, z}\right)^{2}\right)_{s} d z
\end{aligned}
$$

or by (5.3) equivalently

$$
\begin{aligned}
& \llbracket\left[\rho_{\varepsilon}\left(\left(u_{v}\right)_{\varepsilon}-\left(w_{v}\right)_{\varepsilon}\right)\left(\mathbf{u}_{\varepsilon}-\mathbf{w}_{\varepsilon}\right)+p\left(\rho_{\varepsilon}\right) \boldsymbol{v}_{\varepsilon} \rrbracket_{1}=\right. \\
& -\left(w_{0}^{j}\right)_{\tau} \int_{0}^{\infty}\left(R_{0}-\rho_{0}^{+}\right) d z-\int_{-\infty}^{0} \ldots d z \\
& \quad-\int_{0}^{\infty}\left[\frac{1}{\left|\mathbf{t}_{0}\right|}\left(p\left(R_{0}\right) \frac{t_{0}^{j}}{\left|\mathbf{t}_{0}\right|}\right)_{s}-\frac{1}{\left|\mathbf{t}_{0}\right|}\left(p\left(\rho_{0}^{ \pm}\right) \frac{t_{0}^{j}}{\left|\mathbf{t}_{0}\right|}\right)_{s}\right] d z-\int_{-\infty}^{0} \ldots d z \\
& \quad-\gamma \frac{1}{2} \frac{1}{\left|\mathbf{t}_{0}\right|} \int_{-\infty}^{\infty}\left(\frac{t_{0}^{j}}{\left|\mathbf{t}_{0}\right|}\left(R_{0, z}\right)^{2}\right)_{s} d z
\end{aligned}
$$

Because of (7.11) we have

$$
\frac{j_{0}^{2}}{R_{0}}-\frac{j_{0}^{2}}{\rho_{0}^{ \pm}}-\gamma R_{0} R_{0, z z}=p\left(\rho_{0}^{ \pm}\right)-p\left(R_{0}\right)-\frac{\gamma}{2} R_{0, z}^{2}
$$

Inserting this into (7.98) we can, in view of (3.4), identify the surface stress vector $\sigma_{\Gamma}$ as

$$
\sigma_{\Gamma}=\sigma_{\Gamma}^{j}=\left(\int_{0}^{\infty}\left(\frac{j_{0}^{2}}{R_{0}}-\frac{j_{0}^{2}}{\rho_{0}^{+}}+\gamma R_{0, z}^{2}\right) d z+\int_{-\infty}^{0} \ldots d z\right) \frac{t_{0}^{j}}{\left|\mathbf{t}_{0}\right|^{2}}+\mathcal{O}(\varepsilon) .
$$

\section{Proof of Theorem 6.7}

Now we will establish Theorem 6.7.

Proof. To prove the theorem we will compute the first non-vanishing order of

$$
\llbracket j_{\varepsilon} \rrbracket\left\{W^{\prime}\left(\rho_{\varepsilon}\right)+\frac{\left|\mathbf{u}_{\varepsilon}-\mathbf{w}_{\varepsilon}\right|^{2}}{2}\right\}+\left\{j_{\varepsilon}\right\} \llbracket W^{\prime}\left(\rho_{\varepsilon}\right)+\frac{\left|\mathbf{u}_{\varepsilon}-\mathbf{w}_{\varepsilon}\right|^{2}}{2} \rrbracket
$$

by the jump conditions in Theorem 6.4. 
As we see from (6.13) and (6.14) there are no $\mathcal{O}(1)$ contributions in the jump terms in (8.1), so the leading order terms are of $\mathcal{O}(\varepsilon)$. They are given as products of the order $\mathcal{O}(\varepsilon)$-terms in the jumps and the order $\mathcal{O}(1)$-terms in the mean values, i.e., the leading order of (8.1) is given by

$$
\begin{aligned}
& \llbracket \rho_{\varepsilon}\left(\left(u_{v}\right)_{\varepsilon}-\left(w_{\nu}\right)_{\varepsilon}\right) \rrbracket_{1}\left\{W^{\prime}\left(\rho_{0}\right)+\frac{\left|\mathbf{u}_{0}-\mathbf{w}_{0}\right|^{2}}{2}\right\} \\
& +\left\{\rho_{0}\left(u_{\nu 0}-w_{\nu 0}\right)\right\} \llbracket W^{\prime}\left(\rho_{\varepsilon}\right)+\frac{\left|\mathbf{u}_{\varepsilon}-\mathbf{w}_{\varepsilon}\right|^{2}}{2} \rrbracket_{1} .
\end{aligned}
$$

By (7.13) we have $u_{t 0}=w_{t 0}$ such that $u_{0}^{j}-w_{0}^{j}=\left(u_{\nu 0}-w_{\nu 0}\right) v_{0}^{j}$, whence

$$
\left|\mathbf{u}_{0}-\mathbf{w}_{0}\right|^{2}=\left(u_{\nu 0}-w_{\nu 0}\right)^{2} \quad \text { and } \quad\left(u_{0}^{j}-w_{0}^{j}\right) u_{1}^{j}=\left(u_{\nu 0}-w_{\nu 0}\right) u_{\nu 1} .
$$

Hence, (8.2) equals

$$
\begin{aligned}
\llbracket \rho_{\varepsilon}\left(\left(u_{v}\right)_{\varepsilon}-\left(w_{v}\right)_{\varepsilon}\right) \rrbracket_{1}\left\{W^{\prime}\left(\rho_{0}\right)\right. & \left.+\frac{\left|\mathbf{u}_{0}-\mathbf{w}_{0}\right|^{2}}{2}\right\} \\
& +\left\{\rho_{0}\left(u_{v 0}-w_{\nu 0}\right)\right\} \llbracket W^{\prime}\left(\rho_{\varepsilon}\right)+\frac{\left|\left(u_{\nu}\right)_{\varepsilon}-\left(w_{\nu}\right)_{\varepsilon}\right|^{2}}{2} \rrbracket_{1} .
\end{aligned}
$$

Now we use (6.13) and (6.14) to get an equality

$$
\begin{aligned}
& \llbracket \rho_{\varepsilon}\left(\left(u_{\nu}\right)_{\varepsilon}-\left(w_{\nu}\right)_{\varepsilon}\right) \rrbracket_{1}\left\{W^{\prime}\left(\rho_{0}\right)+\frac{\left|\mathbf{u}_{0}-\mathbf{w}_{0}\right|^{2}}{2}\right\} \\
& \quad+\left\{\rho_{0}\left(u_{\nu 0}-w_{\nu 0}\right)\right\} \llbracket W^{\prime}\left(\rho_{\varepsilon}\right)+\frac{\left|\mathbf{u}_{\varepsilon}-\mathbf{w}_{\varepsilon}\right|^{2}}{2} \rrbracket_{1} \\
& =-j_{0}\left(\int_{0}^{\infty} v_{0}^{j}\left(U_{0}^{j}-\left(u_{0}^{j}\right)^{+}\right)_{\tau} d z+\int_{-\infty}^{0} v_{0}^{j}\left(U_{0}^{j}-\left(u_{0}^{j}\right)^{+}\right)_{\tau} d z\right) \\
& -\left(g\left(\rho_{0}^{+}\right)+\frac{j_{0}^{2}}{2\left(\rho_{0}^{+}\right)^{2}}\right) \int_{0}^{\infty}\left(R_{0}-\rho_{0}^{+}\right)_{\tau} d z-\left(g\left(\rho_{0}^{-}\right)+\frac{j_{0}^{2}}{2\left(\rho_{0}^{-}\right)^{2}}\right) \int_{-\infty}^{0}\left(R_{0}-\rho_{0}^{-}\right)_{\tau} d z \\
& -\left(\frac{1}{\left|\mathbf{t}_{0}\right|}\left(\left|\mathbf{t}_{0}\right| w_{t 0}\right)_{s}-\kappa_{0} w_{\nu 0}\right)\left(g\left(\rho_{0}^{+}\right)+\frac{j_{0}^{2}}{2\left(\rho_{0}^{+}\right)^{2}}\right) \int_{0}^{\infty}\left(R_{0}-\rho_{0}^{+}\right) d z \\
& -\left(\frac{1}{\left|\mathbf{t}_{0}\right|}\left(\left|\mathbf{t}_{0}\right| w_{t 0}\right)_{s}-\kappa_{0} w_{\nu 0}\right)\left(g\left(\rho_{0}^{-}\right)+\frac{j_{0}^{2}}{2\left(\rho_{0}^{-}\right)^{2}}\right) \int_{-\infty}^{0}\left(R_{0}-\rho_{0}^{-}\right) d z \\
& -(\lambda+2 \mu) j_{0}^{2} \int_{-\infty}^{\infty}\left(\left(\frac{1}{R_{0}}\right)_{z}\right)^{2} d z .
\end{aligned}
$$

To simplify the notation we define

$$
\begin{aligned}
& A^{ \pm}:=\mp j_{0} \int_{0}^{ \pm \infty} v_{0}^{j}\left(U_{0}^{j}-\left(u_{0}^{j}\right)^{ \pm}\right)_{\tau} d z \mp\left(g\left(\rho_{0}^{ \pm}\right)+\frac{j_{0}^{2}}{2\left(\rho_{0}^{ \pm}\right)^{2}}\right) \int_{0}^{ \pm \infty}\left(R_{0}-\rho_{0}^{ \pm}\right)_{\tau} d z, \\
& B^{ \pm}:=\mp\left(\frac{1}{\left|\mathbf{t}_{0}\right|}\left(\left|\mathbf{t}_{0}\right| w_{t 0}\right)_{s}-\kappa_{0} w_{\nu 0}\right)\left(g\left(\rho_{0}^{ \pm}\right)+\frac{j_{0}^{2}}{2\left(\rho_{0}^{ \pm}\right)^{2}}\right) \int_{0}^{ \pm \infty}\left(R_{0}-\rho_{0}^{ \pm}\right) d z
\end{aligned}
$$


We will first deal with $A^{ \pm}$. Due to (5.4) there exists $c \in \mathbb{R}$, which is independent of $z$ as $\boldsymbol{v}_{0}$ and $\mathbf{t}_{0}$ are independent of $z$, such that

$$
\left(v_{0}^{j}\right)_{\tau}=c t_{0}^{j}
$$

Therefore we get

$$
\begin{aligned}
\int_{0}^{ \pm \infty} v_{0}^{j}\left(U_{0}^{j}-\left(u_{0}^{j}\right)^{ \pm}\right)_{\tau} d z & =\int_{0}^{ \pm \infty}\left(U_{\nu 0}-\left(u_{\nu 0}\right)^{ \pm}\right)_{\tau} d z-\int_{0}^{ \pm \infty}\left(v_{0}^{j}\right)_{\tau}\left(U_{0}^{j}-\left(u_{0}^{j}\right)^{ \pm}\right) d z \\
& =\int_{0}^{ \pm \infty}\left(U_{\nu 0}-\left(u_{\nu 0}\right)^{ \pm}\right)_{\tau} d z-c \int_{0}^{ \pm \infty}\left(t_{0}^{j} U_{0}^{j}-t_{0}^{j}\left(u_{0}^{j}\right)^{ \pm}\right) d z \\
& \stackrel{(7.6)}{=} \int_{0}^{ \pm \infty}\left(\left(U_{\nu 0}-w_{\nu 0}\right)_{\tau}-\left(u_{\nu 0}^{ \pm}-w_{\nu 0}\right)_{\tau}\right) d z
\end{aligned}
$$

Using (7.9) yields

$$
\begin{aligned}
& \left(g\left(\rho_{0}^{ \pm}\right)+\frac{j_{0}^{2}}{2\left(\rho_{0}^{ \pm}\right)^{2}}\right) \int_{0}^{ \pm \infty}\left(R_{0}-\rho_{0}^{ \pm}\right)_{\tau} d z \\
& =\int_{0}^{ \pm \infty}\left(g\left(\rho_{0}^{ \pm}\right)+\frac{j_{0}^{2}}{2\left(\rho_{0}^{ \pm}\right)^{2}}\right)\left(R_{0}-\rho_{0}^{ \pm}\right)_{\tau} d z \\
& =\int_{0}^{ \pm \infty}\left(g\left(R_{0}\right)+\frac{j_{0}^{2}}{2 R_{0}^{2}}-\gamma R_{0, z z}\right) R_{0, \tau}-\left(g\left(\rho_{0}^{ \pm}\right)+\frac{j_{0}^{2}}{2\left(\rho_{0}^{ \pm}\right)^{2}}\right)\left(\rho_{0}^{ \pm}\right)_{\tau} d z .
\end{aligned}
$$

Inserting (8.8) and (8.9) in (8.6) implies

$$
\begin{aligned}
A^{ \pm}= & \mp \int_{0}^{ \pm \infty}\left(j_{0}\left(\frac{j_{0}}{R_{0}}\right)_{\tau}-j_{0}\left(\frac{j_{0}}{\rho_{0}^{ \pm}}\right)_{\tau}\right) d z \\
& \mp \int_{0}^{ \pm \infty}\left(g\left(R_{0}\right)+\frac{j_{0}^{2}}{2 R_{0}^{2}}-\gamma R_{0, z z}\right) R_{0, \tau}-\left(g\left(\rho_{0}^{ \pm}\right)+\frac{j_{0}^{2}}{2\left(\rho_{0}^{ \pm}\right)^{2}}\right)\left(\rho_{0}^{ \pm}\right)_{\tau} d z \\
= & \mp \int_{0}^{ \pm \infty}\left(W\left(R_{0}\right)-W\left(\rho_{0}^{ \pm}\right)+\frac{j_{0}^{2}}{2 R_{0}}-\frac{j_{0}^{2}}{2 \rho_{0}^{ \pm}}\right)_{\tau} d z \pm \gamma \int_{0}^{ \pm \infty} R_{0, z z} R_{0, \tau} d z .
\end{aligned}
$$

We observe

$$
\int_{-\infty}^{\infty} R_{0, z z} R_{0, \tau}=-\int_{-\infty}^{\infty}\left(\frac{1}{2}\left(R_{0, z}\right)^{2}\right)_{\tau} d z
$$

whence (8.10) implies

$$
\begin{aligned}
A^{+}+A^{-}= & -\int_{0}^{\infty}\left(W\left(R_{0}\right)-W\left(\rho_{0}^{+}\right)+\frac{j_{0}^{2}}{2 R_{0}}-\frac{j_{0}^{2}}{2 \rho_{0}^{+}}+\frac{\gamma}{2} R_{0, z}^{2}\right)_{\tau} d z \\
& -\int_{-\infty}^{0}\left(W\left(R_{0}\right)-W\left(\rho_{0}^{-}\right)+\frac{j_{0}^{2}}{2 R_{0}}-\frac{j_{0}^{2}}{2 \rho_{0}^{-}}+\frac{\gamma}{2} R_{0, z}^{2}\right)_{\tau} d z
\end{aligned}
$$

This finishes our calculations for $A^{ \pm}$and we turn to $B^{ \pm}$. 
First we observe that due to (7.9)

$$
\begin{aligned}
& \left(g\left(\rho_{0}^{ \pm}\right)+\frac{j_{0}^{2}}{2\left(\rho_{0}^{ \pm}\right)^{2}}\right) \int_{0}^{ \pm \infty}\left(R_{0}-\rho_{0}^{ \pm}\right) d z \\
& =\int_{0}^{ \pm \infty}\left(g\left(R_{0}\right)+\frac{j_{0}^{2}}{2 R_{0}^{2}}-\gamma R_{0, z z}\right) R_{0}-\left(g\left(\rho_{0}^{ \pm}\right)+\frac{j_{0}^{2}}{2\left(\rho_{0}^{ \pm}\right)^{2}}\right) \rho_{0}^{ \pm} d z \\
& =\int_{0}^{ \pm \infty}\left(p\left(R_{0}\right)+W\left(R_{0}\right)+\frac{j_{0}^{2}}{2 R_{0}}-\gamma R_{0} R_{0, z z}-p\left(\rho_{0}^{ \pm}\right)-W\left(\rho_{0}^{ \pm}\right)-\frac{j_{0}^{2}}{2 \rho_{0}^{ \pm}}\right) d z .
\end{aligned}
$$

From (7.11) and (7.12) we already know

$$
p\left(R_{0}\right)-p\left(\rho_{0}^{ \pm}\right)-\gamma R_{0} R_{0, z z}=-\frac{j_{0}^{2}}{R_{0}}+\frac{j_{0}^{2}}{\rho_{0}^{ \pm}}-\frac{\gamma}{2} R_{0, z}^{2} .
$$

Inserting (8.12) and (8.13) in (8.7) we find

$$
\begin{aligned}
B^{+} & +B^{-}= \\
& -\left(\frac{1}{\left|\mathbf{t}_{0}\right|}\left(\left|\mathbf{t}_{0}\right| w_{t 0}\right)_{s}-\kappa_{0} w_{\nu 0}\right) \int_{0}^{\infty}\left(W\left(R_{0}\right)-W\left(\rho_{0}^{+}\right)-\frac{j_{0}^{2}}{2 R_{0}}+\frac{j_{0}^{2}}{2 \rho_{0}^{+}}-\frac{\gamma}{2} R_{0, z}^{2}\right) d z \\
& -\left(\frac{1}{\left|\mathbf{t}_{0}\right|}\left(\left|\mathbf{t}_{0}\right| w_{t 0}\right)_{s}-\kappa_{0} w_{\nu 0}\right) \int_{-\infty}^{0}\left(W\left(R_{0}\right)-W\left(\rho_{0}^{-}\right)-\frac{j_{0}^{2}}{2 R_{0}}+\frac{j_{0}^{2}}{2 \rho_{0}^{-}}-\frac{\gamma}{2} R_{0, z}^{2}\right) d z
\end{aligned}
$$

Plugging (8.11) and (8.14) in (8.5) we get

$$
\begin{aligned}
& \llbracket \rho_{\varepsilon}\left(\left(u_{v}\right)_{\varepsilon}-\left(w_{\nu}\right)_{\varepsilon}\right) \rrbracket_{1}\left\{W^{\prime}\left(\rho_{0}\right)+\frac{\left|\mathbf{u}_{0}-\mathbf{w}_{0}\right|^{2}}{2}\right\} \\
& +\left\{\rho_{0}\left(\left(u_{\nu 0}-w_{\nu 0}\right)\right\} \llbracket W^{\prime}\left(\rho_{\varepsilon}\right)+\frac{\left|\mathbf{u}_{\varepsilon}-\mathbf{w}_{\varepsilon}\right|^{2}}{2} \rrbracket_{1}\right. \\
& =-\int_{0}^{\infty}\left(W\left(R_{0}\right)-W\left(\rho_{0}^{+}\right)+\frac{j_{0}^{2}}{2 R_{0}}-\frac{j_{0}^{2}}{2 \rho_{0}^{+}}+\frac{\gamma}{2} R_{0, z}^{2}\right)_{\tau} d z \\
& -\int_{-\infty}^{0}\left(W\left(R_{0}\right)-W\left(\rho_{0}^{-}\right)+\frac{j_{0}^{2}}{2 R_{0}}-\frac{j_{0}^{2}}{2 \rho_{0}^{-}}+\frac{\gamma}{2} R_{0, z}^{2}\right)_{\tau} d z \\
& -\left(\frac{1}{\left|\mathbf{t}_{0}\right|}\left(\left|\mathbf{t}_{0}\right| w_{t 0}\right)_{s}-\kappa_{0} w_{\nu 0}\right) \int_{0}^{\infty}\left(W\left(R_{0}\right)-W\left(\rho_{0}^{+}\right)-\frac{j_{0}^{2}}{2 R_{0}}+\frac{j_{0}^{2}}{2 \rho_{0}^{+}}-\frac{\gamma}{2} R_{0, z}^{2}\right) d z \\
& -\left(\frac{1}{\left|\mathbf{t}_{0}\right|}\left(\left|\mathbf{t}_{0}\right| w_{t 0}\right)_{s}-\kappa_{0} w_{\nu 0}\right) \int_{-\infty}^{0}\left(W\left(R_{0}\right)-W\left(\rho_{0}^{-}\right)-\frac{j_{0}^{2}}{2 R_{0}}+\frac{j_{0}^{2}}{2 \rho_{0}^{-}}-\frac{\gamma}{2} R_{0, z}^{2}\right) d z \\
& \quad-(\lambda+2 \mu) j_{0}^{2} \int_{-\infty}^{\infty}\left(\left(\frac{1}{R_{0}}\right)_{z}\right)^{2} d z .
\end{aligned}
$$


Recalling equation (3.10) and definitions (6.17), (6.18) we can identify $W_{\Gamma}$ as

$$
\begin{aligned}
W_{\Gamma}=\int_{0}^{\infty}\left(W\left(R_{0}\right)-W\left(\rho_{0}^{+}\right)+\right. & \left.\frac{1}{2} \frac{j_{0}^{2}}{R_{0}}-\frac{1}{2} \frac{j_{0}^{2}}{\rho_{0}^{+}}+\frac{\gamma}{2} R_{0, z}^{2}\right) d z \\
& +\int_{-\infty}^{0}\left(W\left(R_{0}\right)-W\left(\rho_{0}^{-}\right)+\frac{1}{2} \frac{j_{0}^{2}}{R_{0}}-\frac{1}{2} \frac{j_{0}^{2}}{\rho_{0}^{-}}+\frac{\gamma}{2} R_{0, z}^{2}\right) d z .
\end{aligned}
$$

and obtain the assertion of Theorem 6.7.

\section{Kinetic relation}

We will show that the leading order jump conditions stated in Theorem 6.4 consist of the two conservation laws for mass and momentum and in addition of a kinetic relation. In particular, when the direction of the mass flux is given, the normal velocity of the interface is determined by the state on one side of the interface.

LEMMA 9.1 Let $\rho_{0}^{+}, \rho_{0}^{-}, u_{\nu 0}^{+}, u_{\nu 0}^{-}, u_{t 0}^{+}, u_{t 0}^{-}, w_{\nu 0}$ satisfy the jump conditions from Lemma 7.1 , such that $\rho_{0}^{-} \notin[\alpha, \beta]$, where $\alpha, \beta$ are the Maxwell points of $W$, and the equal area construction

$$
0=-\int_{\frac{1}{\rho_{0}}}^{\frac{1}{\rho_{0}^{+}}} p\left(\rho_{0}\right) d\left(\frac{1}{\rho_{0}}\right)+\left\{p\left(\rho_{0}\right)\right\} \llbracket \frac{1}{\rho_{0}} \rrbracket
$$

is possible. If $\rho_{0}^{-}, u_{\nu 0}^{-}, u_{t 0}^{-}$and the sign of $j_{0}$ are specified, then $\rho_{0}^{+}, u_{\nu 0}^{+}, u_{t 0}^{+}, w_{\nu 0}$ are determined. Proof. Due to (7.3) we know $u_{t 0}^{+}$equals $u_{t 0}^{-}$. Using (7.2) we get the following expression for the mass flux

$$
j_{0}^{2}=-\frac{\llbracket p\left(\rho_{0}\right) \rrbracket}{\llbracket \frac{1}{\rho_{0}} \rrbracket} .
$$

Inserting (9.2) in (7.4) we find

$$
\llbracket g\left(\rho_{0}\right) \rrbracket=-\frac{1}{2} j_{0}^{2} \llbracket\left(\frac{1}{\rho_{0}}\right)^{2} \rrbracket=\frac{1}{2} \llbracket p\left(\rho_{0}\right) \rrbracket \frac{\left(\frac{1}{\rho_{0}^{+}}\right)^{2}-\left(\frac{1}{\rho_{0}^{-}}\right)^{2}}{\frac{1}{\rho_{0}^{+}}-\frac{1}{\rho_{0}^{-}}}=\llbracket p\left(\rho_{0}\right) \rrbracket\left\{\frac{1}{\rho_{0}}\right\} .
$$

This is equivalent to

$$
0=\llbracket g\left(\rho_{0}\right)-\frac{p\left(\rho_{0}\right)}{\rho_{0}} \rrbracket+\left\{p\left(\rho_{0}\right)\right\} \llbracket \frac{1}{\rho_{0}} \rrbracket .
$$

From equation (2.5) we conclude

$$
\frac{d}{d \frac{1}{\rho_{0}}}\left(g\left(\rho_{0}\right)-\frac{p\left(\rho_{0}\right)}{\rho_{0}}\right)=-p\left(\rho_{0}\right) .
$$

Inserting (9.5) in (9.4) we obtain

$$
0=-\int_{\frac{1}{\rho_{0}}}^{\frac{1}{\rho_{0}^{+}}} p\left(\rho_{0}\right) d\left(\frac{1}{\rho_{0}}\right)+\left\{p\left(\rho_{0}\right)\right\} \llbracket \frac{1}{\rho_{0}} \rrbracket .
$$


Note that (9.6) is an equal area rule which determines $\rho_{0}^{+}$. Then (9.2) prescribes $j_{0}$ which we can, in turn, use to obtain $w_{\nu 0}$ and $u_{\nu 0}^{+}$from (7.1).

As shown in [5] the kinetic relation derived in Lemma 9.1 guarantees the solvability of the free boundary value problem for $\rho_{0}, \mathbf{u}_{0}$. Hence, when we want to determine $\rho_{1}^{+}, u_{v 1}^{+}, u_{t 1}^{+}$and $w_{v 1}$ from $\rho_{1}^{-}, u_{\nu 1}^{-}$and $u_{t 1}^{-}$, we can assume that we can, at least numerically, calculate $\rho_{0}, \mathbf{u}_{0}$ and $\mathbf{w}_{0}$. Furthermore $R_{0}$ and $\mathbf{U}_{0}$ are traveling wave solutions of the non-viscous problem. Their phase portraits can be found in [4].

For the following lemma we assume that we have solved, at least numerically, the inner and outer problem of zeroth order. Hence, we know the right hand side of the first order jump conditions in Theorem 6.4.

LEMMA 9.2 Let $\rho_{1}^{+}, \rho_{1}^{-}, u_{v 1}^{+}, u_{v 1}^{-}, u_{t 1}^{+}, u_{t 1}^{-}, w_{\nu 1}$ satisfy the first order of the jump conditions in Theorem 6.4. If $\rho_{1}^{-}, u_{v 1}^{-}, u_{t 1}^{-}$are given and $j_{0} \neq 0$, then $\rho_{1}^{+}, u_{v 1}^{+}, u_{t 1}^{+}, w_{v 1}$ are determined.

Proof. We start by determining $u_{t 1}^{+}$. We multiply (7.77) by $\mathbf{t}_{0}$ and obtain

$$
j_{0} u_{t 1}^{+}=h_{1},
$$

where $h_{1}$ comprises terms depending only on $\rho_{1}^{-}, u_{v 1}^{-}, u_{t 1}^{-}$and terms of zeroth order and their derivatives. As $j_{0} \neq 0$ equation (9.7) prescribes the value of $u_{t 1}^{+}$. To determine $\rho_{1}^{+}, u_{v 1}^{+}, w_{\nu 1}$ we consider (7.58), (7.68) and (7.77) times $\boldsymbol{v}_{0}$. We obtain a system of linear equations for $\rho_{1}^{+}, u_{v 1}^{+}, w_{v 1}$ which reads

$$
\underbrace{\left(\begin{array}{ccc}
\frac{j_{0}}{\rho_{0}^{+}} & \rho_{0}^{+} & -\rho_{0}^{+}+\rho_{0}^{-} \\
g^{\prime}\left(\rho_{0}^{+}\right) & \frac{j_{0}}{\rho_{0}^{+}} & -\frac{j_{0}}{\rho_{0}^{+}}+\frac{j_{0}}{\rho_{0}^{-}} \\
\left(\frac{j_{0}}{\rho_{0}^{+}}\right)^{2}+p^{\prime}\left(\rho_{0}^{+}\right) & 2 j_{0} & 0
\end{array}\right)}_{=: A}\left(\begin{array}{c}
\rho_{1}^{+} \\
u_{v 1}^{+} \\
w_{\nu 1}
\end{array}\right)=\left(\begin{array}{c}
h_{2} \\
h_{3} \\
h_{4}
\end{array}\right),
$$

where $h_{2}, \ldots, h_{4}$ only contain terms depending on $\rho_{1}^{-}, u_{v 1}^{-}, u_{t 1}^{-}$, terms of zeroth order and their derivatives and $r_{1}$. An easy calculation shows

$\operatorname{det} A=\frac{j_{0}}{\rho_{0}^{-}}\left(\rho_{0}^{+}-\rho_{0}^{-}\right)^{2}\left(g^{\prime}\left(\rho_{0}^{+}\right)-\frac{j_{0}^{2}}{\left(\rho_{0}^{+}\right)^{3}}\right)=\frac{j_{0}}{\rho_{0}^{-}\left(\rho_{0}^{+}\right)^{3}}\left(\rho_{0}^{+}-\rho_{0}^{-}\right)^{2}\left(-\frac{\partial p}{\partial\left(\frac{1}{\rho_{0}}\right)}\left(\rho_{0}^{+}\right)+\frac{\llbracket p\left(\rho_{0}\right) \rrbracket}{\llbracket \frac{1}{\rho_{0}} \rrbracket}\right)$.

The last expression vanishes if and only if the tangent at $\rho_{0}^{+}$and the secant connecting $\rho_{0}^{+}$to $\rho_{0}^{-}$ coincide. However this is not possible due to the equal area rule (9.1).

Thus we have a relation $w_{\nu 1}\left(r_{1}\right)$ such that we end up with an ODE to determine the interface position up to the first order.

Acknowledgements. JG and CR would like to thank the German Research Foundation (DFG) for financial support of the project within the Cluster of Excellence in Simulation Technology (EXC 310/1) at the University of Stuttgart.

CK and WD would like to thank the German Research Foundation (DFG) for financial support of the project "Modeling and sharp interface limits of local and non-local generalized Navier-StokesKorteweg Systems". 


\section{A. Appendix: List of the $\varepsilon$-expansions of the jump brackets and proof of Lemma 7.3}

In this section we provide identities for the first two orders of the jump brackets of mass, Gibbs free energy and pressure.

\section{A.1 Mass flux}

We have

$$
\begin{aligned}
& \llbracket \rho_{\varepsilon}\left(\left(u_{v}\right)_{\varepsilon}-\left(w_{v}\right)_{\varepsilon}\right) \rrbracket \\
& =\lim _{z \rightarrow 0, z>0} \rho_{\varepsilon}\left(\mathbf{r}_{\varepsilon}+z \boldsymbol{v}_{\varepsilon}\right)\left(v_{\varepsilon}^{i} u_{\varepsilon}^{i}\left(\mathbf{r}_{\varepsilon}+z \boldsymbol{v}_{\varepsilon}\right)-v_{\varepsilon}^{i} w_{\varepsilon}^{i}\right)- \\
& \lim _{z \rightarrow 0, z<0} \rho_{\varepsilon}\left(\mathbf{r}_{\varepsilon}+z \boldsymbol{v}_{\varepsilon}\right)\left(v_{\varepsilon}^{i} u_{\varepsilon}^{i}\left(\mathbf{r}_{\varepsilon}+z \boldsymbol{v}_{\varepsilon}\right)-v_{\varepsilon}^{i} w_{\varepsilon}^{i}\right) .
\end{aligned}
$$

To determine the first orders of the jump we will look at the $\varepsilon$-expansion of $\rho_{\varepsilon}$ :

$$
\begin{aligned}
& \rho_{\varepsilon}\left(\mathbf{r}_{\varepsilon}+z \boldsymbol{v}_{\varepsilon}\right)\left(v_{\varepsilon}^{i} u_{\varepsilon}^{i}\left(\mathbf{r}_{\varepsilon}+z \boldsymbol{v}_{\varepsilon}\right)-v_{\varepsilon}^{i} w_{\varepsilon}^{i}\right) \\
=\rho_{0}\left(\mathbf{r}_{0}+z \boldsymbol{v}_{0}\right)\left(v_{0}^{i} u_{\varepsilon}^{i}\left(\mathbf{r}_{0}+z \boldsymbol{v}_{0}\right)-v_{0}^{i} w_{0}^{i}\right) & \\
+ & \varepsilon\left[\left(\rho_{1}\left(r_{0}+z v_{0}\right)+\left(\frac{\partial \rho_{0}}{\partial x^{k}}\right)\left(\mathbf{r}_{0}+z \boldsymbol{v}_{0}\right)\left(r_{1}^{k}+z v_{1}^{k}\right)\right)\left(v_{0}^{i} u_{0}^{i}\left(\mathbf{r}_{0}+z \boldsymbol{v}_{0}\right)-v_{0}^{i} w_{0}^{i}\right)\right. \\
& +\rho_{0}\left(\mathbf{r}_{0}+z \boldsymbol{v}_{0}\right)\left(v_{1}^{i} u_{0}^{i}\left(\mathbf{r}_{0}+z \boldsymbol{v}_{0}\right)+v_{0}^{i} u_{1}^{i}\left(\mathbf{r}_{0}+z \boldsymbol{v}_{0}^{i}\right)+v_{0}^{i}\left(\frac{\partial u_{0}^{i}}{\partial x^{k}}\right)\left(\mathbf{r}_{0}+z \boldsymbol{v}_{0}\right)\left(r_{1}^{k}+z v_{1}^{k}\right)\right. \\
& \left.\left.-v_{1}^{i} w_{0}^{i}-v_{0}^{i} w_{1}^{i}\right)\right]+\mathcal{O}\left(\varepsilon^{2}\right)
\end{aligned}
$$

Hence we obtain the following two jump conditions:

$$
\begin{aligned}
\llbracket \rho_{\varepsilon}\left(\left(u_{v}\right)_{\varepsilon}-\left(w_{v}\right)_{\varepsilon}\right) \rrbracket_{0}= & {\left[\rho_{0}\left(u_{v 0}-w_{v 0}\right)\right] } \\
\llbracket \rho_{\varepsilon}\left(\left(u_{v}\right)_{\varepsilon}-\left(w_{v}\right)_{\varepsilon}\right) \rrbracket_{1}= & \left(\rho_{1}^{+}+\left(\frac{\partial \rho_{0}}{\partial x^{k}}\right)^{+} r_{1}^{k}\right)\left(v_{0}^{i}\left(u_{0}^{i}\right)^{+}-v_{0}^{i} w_{0}^{i}\right) \\
& +\rho_{0}^{+}\left(v_{1}^{i}\left(u_{0}^{i}\right)^{+}+v_{0}^{i}\left(u_{1}^{i}\right)^{+}+v_{0}^{i}\left(\frac{\partial u_{0}^{i}}{\partial x^{k}}\right)^{+} r_{1}^{k}-v_{1}^{i} w_{0}^{i}-v_{0}^{i} w_{1}^{i}\right) \\
& -\left(\rho_{1}^{-}+\left(\frac{\partial \rho_{0}}{\partial x^{k}}\right)^{-} r_{1}^{k}\right)\left(v_{0}^{i}\left(u_{0}^{i}\right)^{-}-v_{0}^{i} w_{0}^{i}\right) \\
& -\rho_{0}^{-}\left(v_{1}^{i}\left(u_{0}^{i}\right)^{-}+v_{0}^{i}\left(u_{1}^{i}\right)^{-}+v_{0}^{i}\left(\frac{\partial u_{0}^{i}}{\partial x^{k}}\right)^{-} r_{1}^{k}-v_{1}^{i} w_{0}^{i}-v_{0}^{i} w_{1}^{i}\right)
\end{aligned}
$$




\section{A.2 Balance of Gibbs free energy}

$$
\begin{aligned}
\llbracket \frac{1}{2}\left(\left(u_{\nu}\right)_{\varepsilon}-\left(w_{\nu}\right)_{\varepsilon}\right)^{2}+g\left(\rho_{\varepsilon}\right) \rrbracket_{0}= & \llbracket \frac{1}{2}\left(u_{\nu 0}-w_{\nu 0}\right)^{2}+g\left(\rho_{0}\right) \rrbracket \\
\llbracket \frac{1}{2}\left(\left(u_{\nu}\right)_{\varepsilon}-\left(w_{\nu}\right)_{\varepsilon}\right)^{2}+g\left(\rho_{\varepsilon}\right) \rrbracket_{1}= & -\left(u_{\nu 0}^{+}-w_{\nu 0}\right) w_{\nu 1}+\left(u_{\nu 0}^{-}-w_{\nu 0}\right) w_{\nu 1} \\
& +\left(u_{\nu 0}^{+}-w_{\nu 0}\right)\left(\left(u_{0}^{j}\right)^{+} v_{1}^{j}+\left(u_{1}^{j}\right)^{+} v_{0}^{j}+\left(\frac{\partial u_{0}^{j}}{\partial x^{k}}\right)^{+} v_{0}^{j} r_{1}^{k}\right) \\
& -\left(u_{\nu 0}^{-}-w_{\nu 0}\right)\left(\left(u_{0}^{j}\right)^{-} v_{1}^{j}+\left(u_{1}^{j}\right)^{-} v_{0}^{j}+\left(\frac{\partial u_{0}^{j}}{\partial x^{k}}\right)^{-} v_{0}^{j} r_{1}^{k}\right) \\
& +g^{\prime}\left(\rho_{0}^{+}\right) \rho_{1}^{+}+g^{\prime}\left(\rho_{0}^{+}\right)\left(\frac{\partial \rho_{0}}{\partial x^{k}}\right)^{+} r_{1}^{k} \\
& -g^{\prime}\left(\rho_{0}^{-}\right) \rho_{1}^{-}-g^{\prime}\left(\rho_{0}^{-}\right)\left(\frac{\partial \rho_{0}}{\partial x^{k}}\right)^{-} r_{1}^{k}
\end{aligned}
$$

The derivation of (A.4) and (A.5) is analogous to the derivation of (A.2) and (A.3).

\section{A.3 Momentum balance}

$$
\begin{aligned}
& \left.\llbracket \rho_{\varepsilon}\left(\left(u_{\nu}\right)_{\varepsilon}-\left(w_{\nu}\right)_{\varepsilon}\right)\left(\mathbf{u}_{\varepsilon}-\mathbf{w}_{\varepsilon}\right)+p\left(\rho_{\varepsilon}\right) \boldsymbol{v}_{\varepsilon} \rrbracket_{0}=\llbracket \rho_{0}\left(u_{\nu 0}\right)-w_{\nu 0}\right)\left(\mathbf{u}_{0}-\mathbf{w}_{0}\right)+p\left(\rho_{0}\right) v_{0} \rrbracket \\
& \llbracket \rho_{\varepsilon}\left(\left(u_{v}\right)_{\varepsilon}-\left(w_{\nu}\right)_{\varepsilon}\right)\left(\mathbf{u}_{\varepsilon}-\mathbf{w}_{\varepsilon}\right)+p\left(\rho_{\varepsilon}\right) \boldsymbol{v}_{\varepsilon} \rrbracket_{1} \\
& =2 j_{0} v_{0}^{i}\left(\left(u_{0}^{j}\right)^{+} v_{1}^{j}+\left(u_{1}^{j}\right)^{+} v_{0}^{j}+\left(\frac{\partial u_{0}^{j}}{\partial x^{k}}\right)^{+} v_{0}^{j} r_{1}^{k}\right) \\
& -2 j_{0} v_{0}^{i}\left(\left(u_{0}^{j}\right)^{-} v_{1}^{j}+\left(u_{1}^{j}\right)^{-} v_{0}^{j}+\left(\frac{\partial u_{0}^{j}}{\partial x^{k}}\right)^{-} v_{0}^{j} r_{1}^{k}\right) \\
& +j_{0} t_{0}^{i}\left(\left(u_{1}^{j}\right)^{+} \frac{t_{0}^{j}}{\left|\mathbf{t}_{0}\right|^{2}}+\left(\frac{\partial u_{0}^{j}}{\partial x^{k}}\right)^{+} \frac{t_{0}^{j}}{\left|\mathbf{t}_{0}\right|^{2}} r_{1}^{k}+\left(u_{0}^{k}\right)^{+}\left(\frac{t_{1}^{k}}{\left|\mathbf{t}_{0}\right|^{2}}-2 \frac{t_{0}^{k} t_{0}^{j} t_{1}^{j}}{\left|\mathbf{t}_{0}\right|^{4}}\right)\right) \\
& -j_{0} t_{0}^{i}\left(\left(u_{1}^{j}\right)^{-} \frac{t_{0}^{j}}{\left|\mathbf{t}_{0}\right|^{2}}+\left(\frac{\partial u_{0}^{j}}{\partial x^{k}}\right)^{-} \frac{t_{0}^{j}}{\left|\mathbf{t}_{0}\right|^{2}} r_{1}^{k}+\left(u_{0}^{k}\right)^{-}\left(\frac{t_{1}^{k}}{\left|\mathbf{t}_{0}\right|^{2}}-2 \frac{t_{0}^{k} t_{0}^{j} t_{1}^{j}}{\left|\mathbf{t}_{0}\right|^{4}}\right)\right) \\
& +v_{0}^{i}\left(\frac{j_{0}^{2}}{\left(\rho_{0}^{+}\right)^{2}}+p^{\prime}\left(\rho_{0}^{+}\right)\right)\left(\rho_{1}^{+}+\left(\frac{\partial \rho_{0}}{\partial x^{k}}\right)^{+} r_{1}^{k}\right) \\
& -v_{0}^{i}\left(\frac{j_{0}^{2}}{\left(\rho_{0}^{-}\right)^{2}}+p^{\prime}\left(\rho_{0}^{-}\right)\right)\left(\rho_{1}^{-}+\left(\frac{\partial \rho_{0}}{\partial x^{k}}\right)^{-} r_{1}^{k}\right)
\end{aligned}
$$

The derivation of (A.6) and (A.7) is analogous to the derivation of (A.2) and (A.3). 


\section{REFERENCES}

1. Alt, H.W. The entropy principle for interfaces. Fluids and solids. Adv. Math. Sci. Appl. 19 (2009) 585663. Zbl1194.35003 MR2606135

2. Anderson, D. M., McFadden, G. B., \& Wheeler, A. A. Diffuse-interface methods in fluid mechanics. Ann. Rev. Fluid Mech. 30 (1998), 139-165. MR1609626

3. Bedjaoui, N. \& LeFloch, P. G. Diffusive-dispersive traveling waves and kinetic relations. IV. Compressible Euler equations. Chinese Ann. Math. Ser. B 24 (2003), 17-34. Zbl1033. 35074 MR1966594

4. Benzoni-Gavage, S., Danchin, R., Descombes, S. \& Jamet, D. Stability issues in the EulerKorteweg model. Contemp. Math. 426 (2007), 103-127. Zbl1119.76029 MR2311523

5. Benzoni-Gavage, S. \& FreistüHLER, H. Effects of surface tension on the stability of dynamical liquid-vapor interfaces. Arch. Ration. Mech. Anal. 174 (2004), 111-150. Zbl1081. 76027 MR2092998

6. Bresch, D., Desjardins, B. \& Lin, C. K. On some compressible fluid models: Korteweg, lubrication, and shallow water systems. Comm. Partial Differential Equations 28 (2003), 843-868. Zbl 106.76436 MR1978317

7. Caginalp, G. \& Fife, P. C. Dynamics of layered interfaces arising from phase boundaries. SIAM J. Appl. Math. 48 (1988), 506-518. MR0941098

8. DANChin, R. \& DesJaRdins, B. Existence of solutions for compressible fluid models of Korteweg type. Ann. Inst. H. Poincaré Anal. Non Linéaire 18 (2001), 97-133. Zb11010 .76075 MR1810272

9. DREYER W. \& Kraus C. The sharp interface limit of the van der Waals-Cahn-Hilliard phase model for fixed and time dependent domains. Proc. R. Soc. A 140 A (2010), 1-26.

10. DREYER W. On jump conditions at phase boundaries for ordered and disordered phases, WIAS preprint no. 869, www.wias-berlin.de/publications/wias-publ/run.jsp? template=abstract\&type=Preprint\&year $=2003 \&$ mumber $=869$

11. FeIREISL, E. Compressible Navier-Stokes equations with a non-monotone pressure law. J. Differential Equations 184 (2002), 97-108. Zbl1012.76079 MR1929148

12. Gaskell, D. R. Chapter 5 - Metallurgical thermodynamics, In: Cahn R. W. and Hansen P., Editors, Physical Metallurgy (Fourth edition) 413-469, North-Holland, Oxford (1996)

13. Gel'Fand, I. M. \& Shilov, G. E. Generalized Functions. Vol. 1, Properties and operations, Translated from the Russian by Eugene Saletan, Academic Press 1964. MR0435831

14. Hattori, H. \& Li, D. The existence of global solutions to a fluid dynamic model for materials for Korteweg type. J. Partial Differential Equations 9 (1996), 323-342. Zb10881. 35095 MR1426082

15. Hermsdörfer, K., Kraus, C. \& Kröner, D. Interface conditions for limits of the Navier-StokesKorteweg model. Interfaces Free Bound. 13 (2011), 239-254. Zbl1219. 35191 MR2813525

16. Jacobs, D., McKinney, B. \& Shearer, M. Travelling wave solutions of the modified Korteweg-de Vries-Burgers equation. J. Differential Equations 116 (1995), 448-467. Zb10820.35118 MR1318583

17. Klainerman, S. \& Majda, A. Compressible and incompressible fluids. Comm. Pure Appl. Math. 35 (1982), 629-651. Zb10478.76091 MR0668409

18. KLEIN, R. Semi-implicit extension of a Godunov-type scheme based on low Mach number asymptotics. I. One-dimensional flow. J. Comput. Phys. 121 (1995) 213-237. Zb10842. 76053 MR1354300

19. Котsсноте, M. Strong solutions for a compressible fluid model of Korteweg type. Ann. Inst. H. Poincaré Anal. Non Linéaire 25 (2008), 679-696. Zb11141. 76053 MR2436788

20. Luckhaus, S. \& ModicA, L. The Gibbs-Thompson relation within the gradient theory of phase transitions. Arch. Rational Mech. Anal. 107 (1989), 71-83. Zb10681. 49012 MR1000224

21. ModicA, L. The gradient theory of phase transitions and the minimal interface criterion. Arch. Rational Mech. Anal. 98 (1987), 123-142. Zbl0616.76004 MR0866718

22. Munz, C. D., Dumbser, M. \& Zucchini, M. The multiple pressure variables method for fluid dynamics and aeroacoustics at low Mach numbers, In: Numerical methods for hyperbolic and kinetic 
problems, volume 7 of IRMA Lect. Math. Theor. Phys. 335-359, Eur. Math. Soc., Zürich (2005). Zb11210.76136 MR2186377

23. TRuskinovsky, L. About the "normal growth" approximation in the dynamical theory of phase transitions. Contin. Mech. Thermodyn. 6 (1994), 185-208. Zb10877.73006 MR1285921 